Schilcher, Anita; Binder, Karin; Krauss, Stefan; Schulte, Magdalena; Rank, Astrid; Deml, Isabell; Hilbert, Sven

\title{
FaWi-S - Eine psychometrische Testkonstruktion zum didaktischen Wissen von Grundschullehrkräften in Bezug auf Sprachförderung im Projekt
}

\section{Eva-Prim}

Mackowiak, Katja [Hrsg.]; Beckerle, Christine [Hrsg.]; Gentrup, Sarah [Hrsg.]; Titz, Cora [Hrsg.]: Forschungsinstrumente im Kontext institutioneller (schrift-)sprachlicher Bildung. Bad Heilbrunn : Verlag Julius Klinkhardt 2020, S. 33-53

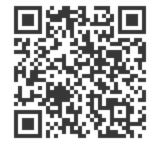

Quellenangabe/ Reference:

Schilcher, Anita; Binder, Karin; Krauss, Stefan; Schulte, Magdalena; Rank, Astrid; Deml, Isabell; Hilbert, Sven: FaWi-S - Eine psychometrische Testkonstruktion zum didaktischen Wissen von Grundschullehrkräften in Bezug auf Sprachförderung im Projekt Eva-Prim - In: Mackowiak, Katja [Hrsg.]; Beckerle, Christine [Hrsg.]; Gentrup, Sarah [Hrsg.]; Titz, Cora [Hrsg.]: Forschungsinstrumente im Kontext institutioneller (schrift-)sprachlicher Bildung. Bad Heilbrunn : Verlag Julius Klinkhardt 2020, S. 33-53 - URN: urn:nbn:de:0111-pedocs-201434 - DOI: 10.25656/01:20143

https://nbn-resolving.org/urn:nbn:de:0111-pedocs-201434 https://doi.org/10.25656/01:20143

in Kooperation mit / in cooperation with:

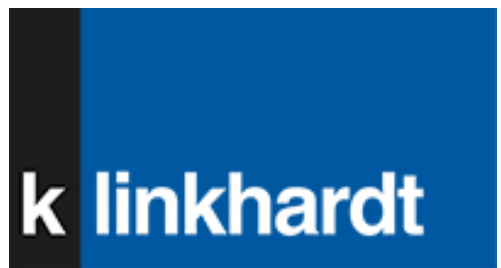

http://www.klinkhardt.de

\section{Nutzungsbedingungen}

Dieses Dokument steht unter folgender Creative Commons-Lizenz: http://creativecommons.org/licenses/by-nc-sa/4.0/deed.de - Sie dürfen das Werk bzw. den Inhalt unter folgenden Bedingungen vervielfältigen, verbreiten und öffentlich zugänglich machen sowie Abwandlungen und Bearbeitungen des Werkes bzw. Inhaltes anfertigen: Sie müssen den Namen des Autors/Rechteinhabers in der von ihm festgelegten Weise nennen. Dieses Werk bzw. der Inhalt darf nicht für kommerzielle Žwecke verwendet werden. Die neu entstandenen Werke bzw. Inhalte dürfen nur unter Verwendung von Lizenzbedingungen weitergegeben werden, die mit denen dieses Lizenzvertrages identisch oder vergleichbar sind.

Mit der Verwendung dieses Dokuments erkennen Sie die Nutzungsbedingungen an.

\section{Terms of use}

This document is published under following Creative Commons-License: http://creativecommons.org/licenses/by-nc-sa/4.0/deed.en - You may copy, distribute and transmit, adapt or exhibit the work in the public and alter, transform or change this work as long as you attribute the work in the manner specified by the author or licensor. You are not allowed to make commercial use of the work. If you alter, transform, or change this work in any way, you may distribute the resulting work only under this or a comparable license.

By using this particular document, you accept the above-stated conditions of use.

\section{Kontakt / Contact:}

\section{peDOCs}

DIPF | Leibniz-Institut für Bildungsforschung und Bildungsinformation Informationszentrum (IZ) Bildung

E-Mail: pedocs@dipf.de

Internet: www.pedocs.de

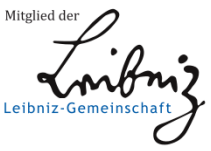


Anita Schilcher, Karin Binder, Stefan Krauss, Magdalena Schulte, Astrid Rank, Isabell Deml und Sven Hilbert

\section{FaWi-S - Eine psychometrische Testkonstruktion zum didaktischen Wissen von Grundschullehrkräften in Bezug auf Sprachförderung im Projekt Eva-Prim}

\section{Info-Kasten zum Instrument}

$\begin{array}{ll}\text { Name } & \begin{array}{l}\text { Professionswissenstest FaWi-S } \\ \text { Autor*innen }\end{array} \\ & \begin{array}{l}\text { Magdalena Schulte, Alexandra Merkert, Anita Schilcher, } \\ \text { Lena Bien-Miller, Gerlinde Lenske, Anja Wildemann, Karin Binder, } \\ \text { Astrid Rank und Isabell Deml }\end{array} \\ \text { Ziel } & \begin{array}{l}\text { Erfassung des sprachförderlichen (fachdidaktischen, Fach- und } \\ \text { pädagogischen) Wissens von Grundschullehrkräften }\end{array} \\ \text { Einsatzbereich } & \begin{array}{l}\text { Grundschule } \\ \text { Erhebung }\end{array} \\ \text { Auswertung } & \begin{array}{l}\text { Paper \& Pencil-Test } \\ \text { Quantitatives und qualitatives Kategoriensystem }\end{array}\end{array}$

\section{Einleitung}

Im vorliegenden Beitrag wird ein im Rahmen des Evaluationsprojektes Eva-Prim neu konstruierter Test zum Sprachförderwissen („FaWi-S“) von Grundschullehrkräften vorgestellt. Eva-Prim ${ }^{1}$ („Evaluation in der Primarstufe“) ist ein BiSS-Projekt, in dem von 2015 bis 2018 längsschnittlich an insgesamt drei Messzeitpunkten Grundschullehrkräfte aus vier Bundesländern (Sachsen-Anhalt (V1), Bayern (V2), Baden-Württemberg (V3) und Nordrhein-Westfalen (V4)) sowie deren Schüler*innen (in der 2., 3. und 4. Klasse) zu Aspekten der Sprachförderung im Mathematikunterricht untersucht wurden (Rank et al., im Druck).

Eva-Prim war dabei auf den Mathematikunterricht der Grundschule fokussiert. Das bedeutet, dass im Sinne des mittlerweile einschlägigen Themen- und For-

1 Eva-Prim wurde unter dem Förderkennzeichen $01 \mathrm{Jl}$ 1503A von Bund und Ländern aus Mitteln des Bundesministeriums für Bildung und Forschung gefördert und als Kooperationsprojekt von den Universitäten Landau und Regensburg durchgeführt. 
schungsfeldes „Sprache im Fach“ (z.B. Becker-Mrotzek, Schramm, Thürmann \& Vollmer, 2013) nicht primär das sprachförderliche Handeln der Lehrkräfte im Deutsch-, sondern im Fachunterricht untersucht wurde (Schulte, in Vorb.) und ein Zielkriterium u.a. die sprachliche Ausdrucksfähigkeit der Kinder im Mathematikunterricht war (Merkert, in Vorb.).

Im vorliegenden Beitrag sollen Konzeption und Konstruktion des FaWi-S vorgestellt werden, der sprachförderliche Kompetenzen von (Grundschul-)Lehrkräften unabhängig vom Fach adressiert. Neben den üblichen psychometrischen Gütekriterien sowie einer konfirmatorischen Faktorenanalyse soll dabei im Besonderen betrachtet werden, ob dieses Wissen in der Ausbildung erworben wird und ob - ganz im Sinne des Begriffs „Professionswissen“ bzw. „Expertise“ (Krauss \& Bruckmaier, 2014; Zlatkin-Troitschanskaia, Beck, Sembill, Nickolaus \& Mulder, 2009; Voss, Kunina-Habenicht, Hoehne \& Kunter, 2015) - praktizierende Grundschullehrkräfte erwartungsgemäß besser abschneiden als Lehramtsstudierende. Entsprechende Hinweise auf die Konstruktvalidität würden nicht nur den zukünftigen Einsatz dieses Tests zur Untersuchung der prädiktiven Validität im Hinblick auf Unterrichtsqualität und das Lernen von Schüler*innen im Rahmen von Eva-Prim rechtfertigen, sondern auch die zielgerichtete Implementation durch Forscher*innen mit vergleichbaren Fragestellungen ermöglichen (siehe Online-Anhang).

\section{Theoretischer Hintergrund}

\subsection{Sprache im Fach}

Das Projekt Eva-Prim fokussierte das sprachförderliche Handeln von Lehrkräften im Mathematikunterricht. Auf einen engen Zusammenhang zwischen Lesekompetenz und der Mathematikleistung weisen bereits Large-Scale-Studien wie PISA, IGLU und TIMSS hin (Bos et al., 2012; Leutner, Klieme, Meyer \& Wirth, 2004). Inzwischen wurden die entsprechenden mathematikdidaktischen Fragestellungen auf sprachliche Prozesse im Mathematikunterricht allgemein ausgeweitet (für einen Überblick siehe Schilcher, Röhrl \& Krauss, 2017). Besonders umfassend hat sich vor allem Prediger diesem Themenfeld gewidmet und durch ihre empirischen Studien die Bedeutung der Sprache für Lernen und Verstehen im Mathematikunterricht nachgewiesen. Prediger konnte zeigen, dass gerade die Verflechtung sprachlicher und fachlicher Aspekte entscheidend ist, damit Lernende ihr Wissen strukturieren, erweitern und ein konzeptuelles Verständnis entwickeln können (z.B. Prediger, 2013; Prediger, Renk, Büchter, Gürsoy \& Benholz, 2013; Prediger, Wilhelm, Büchter, Benholz \& Gürsoy, 2015). Dass der Sprachstand für Mathematikaufgaben, die ein konzeptionell-inhaltliches Verstehen erfordern, sogar 
eine größere Bedeutung als die kognitiven Grundfähigkeiten hat, zeigt das Projekt SOKKE (Sozialisation und Akkulturation in Erfahrungsräumen von Kindern mit Migrationshintergrund; Ufer, Reiss \& Mehringer, 2013).

Ein wichtiger Perspektivwechsel in der Mathematikdidaktik erfolgte dabei durch die Fokusverschiebung von der mathematischen Fachsprache und den mathematischen Fachbegriffen hin zu einer, auf allgemeinsprachlichen Kompetenzen aufbauenden, mathematischen Bildungssprache. So konnten Bochnik und Ufer (2016) zeigen, dass zwar mathematisch-fachsprachliche Kompetenzen eine Rolle spielen, vor allem aber allgemeinsprachliche Kenntnisse mathematische Leistungsunterschiede erklären können. Dabei müssen vor allem die syntaktische und textuelle Ebene in den Blick genommen werden, die gerade bei mathematischer Fachsprache durch eine hohe Verdichtung geprägt sind und damit die Kriterien der Bildungssprache (Gogolin, 2008) besonders symptomatisch erfüllen.

Für einen sprachsensiblen Mathematikunterricht scheinen sich derzeit zwei dominante Zugangsweisen herauszukristallisieren. Zum einen sollten Lehrkräfte für die sprachliche Qualität ihrer Erklärungen sensibilisiert und befähigt werden, neuartige Erklärpraxen in ihrem Unterricht zu implementieren (Müller-Hill, 2016; Papadopoulou \& Bescherer, 2016). Zum anderen sollten sie Lernumgebungen so an die (sprachlichen) Bedürfnisse der Lernenden anpassen können, dass sie das Verstehen der Schüler*innen unterstützen (z.B. Hagena, Leiss, Neumann \& Schwippert, 2015; Prediger, 2015). Beide Strategien erfordern von Seiten der Lehrkräfte hohes Sprachförderwissen, das sie befähigt, die eingesetzten Texte und Materialien vor allem im Hinblick auf die Textschwierigkeit zu beurteilen. Dazu gehören Aspekte wie die Wort- und Satzkomplexität, aber auch die Analyse der textuellen Kohärenz und das Erkennen sprachlicher Verständnisbarrieren wie mehrdeutige oder metaphorische Ausdrücke (Wild \& Schilcher, 2019). Um ihre Erklärungen adaptiv an das sprachliche Niveau der Schüler*innen anpassen zu können, müssen die Lehrkräfte fähig sein, ihre Wortwahl auf die Adressat*innen abzustimmen und die Satzkomplexität durch grammatische, aber auch sprecherische Mittel wie die Intonation zu reduzieren. Sie müssen aber auch über spezifische Strategien wie Scaffolding oder sprachliches Modellieren verfügen (Gaier \& Schilcher, in Vorb.; Schilcher, Krauss, Lindl \& Hilbert, in Vorb.). Diese Fähigkeiten wiederum fußen auf einem soliden diagnostischen Wissen, das sich auf die verschiedenen sprachlichen Kompetenzen der Schüler*innen bezieht, etwa Lese-, Rechtschreib- und Schreibdiagnostik (siehe den Überblick von Südkamp \& Praetorius, 2017).

\subsection{Professionswissen von Lehrkräften und dessen Bedeutung für den Lernerfolg von Schüler*innen}

Das professionelle Wissen von Lehrkräften ist gerade in Deutschland seit ca. 15 Jahren ein vieldiskutiertes Thema (z.B. Krauss, Lindl, Schilcher \& Tepner, 2017; Voss et al., 2015), da vor allem seit dem „PISA-Schock“ 2000 nicht nur Kompe- 
tenzen von Schüler*innen, sondern auch von Lehrkräften umfassend empirisch untersucht werden (z.B. COACTIV 03/04; Kunter et al., 2011). Im deutschsprachigen Raum wurden seitdem zahlreiche Testkonstruktionen vorgelegt, die insbesondere die Professionswissensbereiche Fachwissen (FW), Fachdidaktisches Wissen (FDW) und Pädagogisches Wissen (PW) adressieren. Der überwiegende Teil dieser Testkonstruktionen, die im Sinne der Lehrerforschung dem Expertenparadigma zuzurechnen sind (Krauss \& Bruckmaier, 2014), rekurriert dabei theoretisch auf die Professionswissenstaxonomie von Shulman (1986, 1987). Im deutschsprachigen Diskurs haben sich daraus mittlerweile die drei oben genannten Kernkategorien des Lehrerwissens etabliert (s.a. Großprojekte wie TEDS, z.B. Blömeke et al., 2011; ProwiN, z.B. Kirschner et al., 2017; FALKO, z.B. Krauss et al., 2017).

Dieses Wissen spielt für die Unterrichtsqualität und die Lernzuwächse von Schüler*innen eine entscheidende Rolle. In der COACTIV-Studie, in der Mathematiklehrkräfte von PISA-Klassen umfassend befragt wurden, hatte das gemessene fachdidaktische Wissen (FDW) den unter allen betrachteten Lehrerkompetenzen mit Abstand größten nachweisbaren Effekt auf die Unterrichtsqualität und den Lernzuwachs von Schüler*innen (z.B. Kunter et al., 2013). So verzeichneten Klassen, deren Lehrkraft im FDW-Test eine Standardabweichung über dem Mittelwert lag, innerhalb eines Jahres einen durchschnittlichen Lernzuwachs von ungefähr $d=0.5$, während Klassen, die von einer Lehrkraft mit einem FDWScore von einer Standardabweichung unter dem Mittelwert unterrichtet wurden, im selben Zeitraum im Schnitt lediglich einen Lernzuwachs von ca. $d=0.2$ erzielten. Die Differenz von $d=0.3$ entspricht bemerkenswerterweise in etwa der Lernrate eines ganzen Schuljahres (Kunter et al., 2011). Auch in Bezug auf USGrundschüler*innen konstatierten Hill, Rowan und Ball (2005) prädiktive Validität des mathematikdidaktischen Wissens der Lehrkräfte für die entsprechenden Schülerleistungen in Mathematik.

Für den Grundschulbereich gibt es bislang nur vergleichsweise wenige Professionswissenstests (nur sechs von etwa 40 bislang entwickelten Tests haben Grundschullehrkräfte als Zielpopulation, Krauss et al., 2017). Auch spezifische Ergebnisse zum Einfluss des Fachwissens und fachdidaktischen Wissens von Deutschlehrkräften auf Schülerleistungen liegen bislang noch nicht vor, auch wenn es bereits vereinzelte Tests gibt (z.B. Blömeke et al., 2011; Pissarek \& Schilcher, 2017; Riegler \& Wiprächtiger-Geppert, 2016). Untersuchungen zur prädiktiven Validität von pädagogischem Wissen finden sich zum Beispiel bei Lenske et al. (2016). 


\section{Testkonstruktion des FaWi-S}

\subsection{Konzeptualisierung des sprachförderlichen Wissens}

Da bislang kein Professionswissenstest für den Primarbereich existiert, der das (überfachliche) Sprachförderwissen fokussiert, wurde zur Beantwortung der Forschungsfragen von Eva-Prim ein Wissenstest zum sprachförderlichen Wissen (FaWi-S) entwickelt, dessen Konstruktion und (erste) Validierung im vorliegenden Beitrag vorgestellt werden. Ziel ist es, durch den FaWi-S das fachdidaktische Wissen von Lehrkräften der Primarstufe im Hinblick auf Aspekte abzubilden, die für eine sprachförderliche Unterstützung von Schüler*innen als besonders zentral gelten. Dabei soll „sprachförderliches Wissen“ so konzeptualisiert werden, dass die Sprachförderung auf alle Fächer bezogen gesehen wird. Unter „Sprachförderwissen" soll im Folgenden also auch dezidiert sprachdidaktisches Wissen verstanden werden, das sich beispielsweise auf die Inhalte des Fachunterrichts (wie Texte, Themen, Fachwortschatz) bezieht (zum heuristischen Hintergrundmodell, das der Testkonstruktion zugrunde lag, siehe Abb. 1).

Auch wenn Sprachförderwissen in der vorliegenden Testkonstruktion überfachlich gedacht wird, ist die Deutschdidaktik, genauer die Sprachdidaktik, die theoretische Referenzdisziplin, die Impulse für eine solche Testkonstruktion geben kann, da hier bereits einschlägige Sprachförderkonzepte existieren. Traditionell umfasst die Sprachdidaktik dabei die Schreibdidaktik, die Rechtschreibdidaktik, die Mündlichkeitsdidaktik und den Grammatikunterricht. In den letzten Jahren beschäftigen sich Sprachdidaktiker*innen auch zunehmend mit der empirisch ausgerichteten Lesedidaktik, was sich auch in verschiedenen BiSS-Projekten widerspiegelt (z.B. Projekt EILe ${ }^{2}$ ). Auch im Rahmen des FaWi-S soll das Wissen zu pragmatischen Textsorten berücksichtigt werden.

Insgesamt orientiert sich der FaWi-S theoretisch an der neueren Auffassung von Grammatikunterricht als funktionaler und integrativer Sprachförderung, wie ihn auch die Bildungsstandards verorten (KMK, 2004b). Grammatisches Wissen soll damit im Hinblick auf konkrete Anwendungsbereiche erworben werden, in denen die Funktion der jeweils erforderlichen sprachlichen Struktur deutlich wird. Die KMK folgt damit sprachdidaktischen Theoriebildungen, die mit wechselnden Schwerpunktsetzungen seit der kommunikativen Wende diskutiert werden (z.B. Feilke \& Tophinke, 2016). Dabei betonen funktionale Modelle stets die Ausrichtung an den Erfordernissen einer gezielten Sprachförderung (Bredel, 2007).

Darüber hinaus wurde versucht, mit dem FaWi-S auch bewährte überfachliche und bereits von Shulman $(1986,1987)$ beschriebene FDW-Facetten wie „Erklären \& Repräsentieren fachlicher Inhalte“, „fachbezogene Schülerkognitionen

2 Evaluation der Implementation von Konzepten zur Leseförderung in der Sekundarstufe I im Rahmen von BiSS-Verbundprojekten (EILe). 
und Fehlvorstellungen“ sowie - darüber hinaus - „Potential von Lernmaterialien“ abzudecken (siehe Tab. 1). Die erste Facette wurde jedoch an die Erfordernisse der Sprachförderung angepasst, die ja keinen Inhalt an sich darstellt, sondern die Art und Weise, wie Inhalte bearbeitet werden. Deshalb spielen hier Unterstützungsmethoden, Strategien und die Analyse von sprachlichen Darstellungen eine zentrale Rolle. Die zweite und dritte Facette werden wie folgt ausdifferenziert: Neben dem Wissen über typische Schülerfehler spielen die konkrete (Einzelfall-) Diagnostik und das Wissen über spezifische Lernvoraussetzungen eine Rolle, statt des Wissens über das Potenzial von Materialien wird Wissen über schwierigkeitsgenerierende Merkmale von Texten und Aufgaben getestet. Theoretischen und empirischen Arbeiten zum starken Zusammenhang der drei übergeordneten Professionswissensbereiche FW, FDW und PW folgend (z.B. König et al., 2017) sollen im FaWi-S weiterhin Aspekte berücksichtigt werden, die tendenziell eher fachlicher (FW) bzw. pädagogischer Natur (PW) sind, aber einen starken Bezug zur Sprachförderung haben (siehe Abb. 1; Tab. 1).

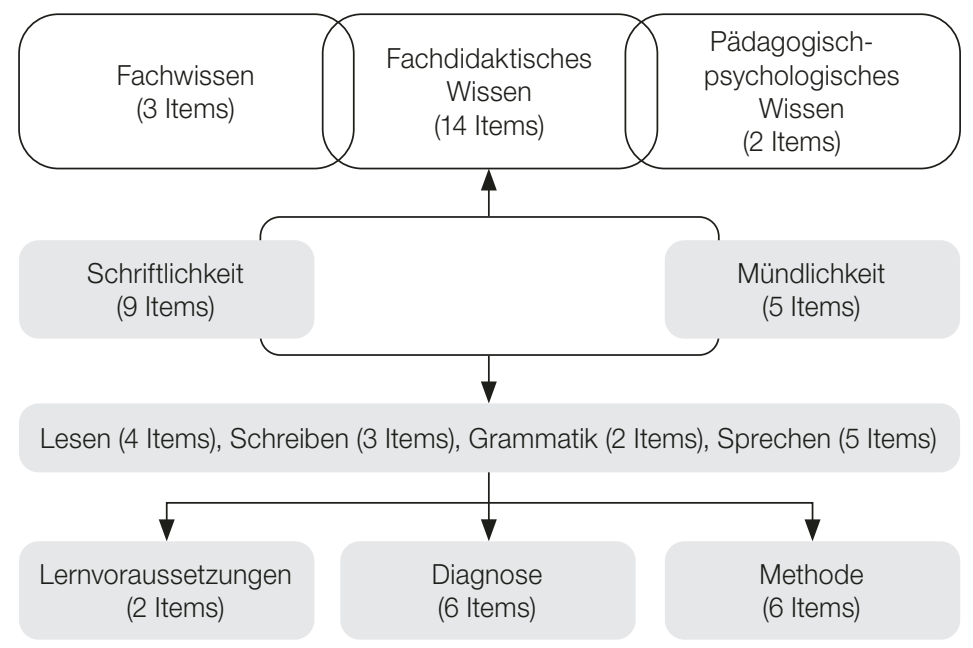

Abb. 1: Einbettung der Items in das heuristische Hintergrundmodell

\subsection{Operationalisierung des sprachförderlichen Wissens}

Der derzeit weit verbreiteten Vorgehensweise bei Paper \& Pencil-Wissenstests folgend (z.B. Krauss et al., 2017) wurden zu den in Abbildung 1 aufgeführten theoretischen Bereichen jeweils Textvignetten entwickelt, die sprachförderliche Aspekte aufgreifen bzw. diesbezüglich didaktisch kritische Unterrichtsszenarien thematisieren (siehe Online-Anhang). Die Konstruktion der Items orientierte sich am aktuellen fachdidaktischen Forschungsstand. Dabei wurde vor allem auf 
Quellen zurückgegriffen, die ihre Thesen durch empirische Forschungsergebnisse absichern können. Wo dies nicht möglich war - weil noch zu wenige gesicherte empirische Ergebnisse vorliegen -, wurde auf normative Vorgaben wie etwa die Bildungsstandards oder Lehrpläne zurückgegriffen (etwa bei der konstruktiven Unterstützung von Schüler*innen bei mündlichen Referaten). Weiterhin lagen aus anderen Projekten bereits Instrumente vor, etwa der Professionswissenstest FALKO-D (Pissarek \& Schilcher, 2017), ein Test zur Messung des fachdidaktischen Wissens von Deutschlehrkräften der Sekundarstufe, sowie der Test aus dem Projekt RESTLESS (Goldenstein, in Vorb.), (alle 19 Items finden sich im OnlineAnhang ebenso wie die Quellen, denen diese entstammen).

Abbildung 1 illustriert die Verteilung der konstruierten Items auf die Facetten des heuristischen Hintergrundmodells. Tabelle 1 schließlich ordnet die konkreten 19 Items des FaWi-S der oben beschriebenen Aufteilung des fachdidaktischen Wissens in „E\&R“ (Wissen über Erklären und Repräsentieren), „SchK“ (Wissen über fachbezogene Schülerkognitionen und typische Fehlvorstellungen) und „Pot“ (Wissen über das Lernpotenzial von Materialien) zu. Die ersten beiden Facetten gehen auf Shulman $(1986,1987)$ zurück, die dritte Facette lehnt sich an die in COACTIV (Kunter et al., 2011) und den sechs FALKO-Disziplinen (Krauss et al., 2017) umgesetzte „Pot“-Facette an. Weiterhin werden in dieser Tabelle die jeweiligen Lernbereiche sowie das Itemformat benannt (für die zwei offenen Items wurde ein Kodiermanual entwickelt). Aus diesen beiden Überblicken wird deutlich, dass es sich um heuristische Strukturierungen handelt, die die deutschdidaktischen Lernbereiche auf die Fachdidaktik-Facetten der COACTIV-Studie beziehen. Items, die mehrere Aspekte tangieren, wurden dabei jeweils dem dominantesten Aspekt zugeordnet. Abbildung 1 und Tabelle 1 dienten demnach als „theoretischer Ideengeber“ zur Itemkonstruktion und zur besseren Orientierung ohne den Anspruch, entsprechende Subfacetten auch jeweils reliabel abzubilden. 
Tab. 1: Übersicht über die Verteilung der FaWi-S-Items auf drei Facetten didaktischen Wissens (FDW) bzw. auf Fachwissen (FW) und pädagogisches Wissen (PW) sowie Zuordnung zu Lernbereichen und ggfs. Deutsch als Zweitsprache (DaZ).

\begin{tabular}{|c|c|c|c|}
\hline $\begin{array}{l}\text { Wissensbereich } \\
\text { (Itemzahl) }\end{array}$ & Item & Lernbereich & $\begin{array}{l}\text { Item- } \\
\text { format }\end{array}$ \\
\hline $\begin{array}{l}\text { FDW-Facette } \\
\text { „E\&R“ (5): } \\
\text { Erklären und Re- } \\
\text { präsentieren von } \\
\text { Fachinhalten }\end{array}$ & $\begin{array}{l}2 \mathrm{~b} \text { „Hilfe für Lesever- } \\
\text { stehen“ } \\
3 \text { „Lesestrategien“ } \\
4 \text { „Dhakiya“ } \\
14 \text { „Simon“ } \\
16 \text { „Unterstützung } \\
\text { Kurzvortrag“ }\end{array}$ & $\begin{array}{l}\text { Lesen } \\
\text { Lesen } \\
\text { Grammatik und DaZ } \\
\text { Mündlichkeit } \\
\text { Mündlichkeit }\end{array}$ & $\begin{array}{l}\text { geschlossen } \\
\text { offen } \\
\text { geschlossen } \\
\text { geschlossen } \\
\text { geschlossen }\end{array}$ \\
\hline $\begin{array}{l}\text { FDW-Facette } \\
\text { „SchK“ (7): } \\
\text { Fachbezogen } \\
\text { Schülerkognitio- } \\
\text { nen und Schwie- } \\
\text { rigkeiten }\end{array}$ & $\begin{array}{l}1 \text { "Orthographie“ } \\
7 \text { „Serda“" } \\
8 \text { "Anne“ } \\
9 \mathrm{a} \text { „sprachliche Struk- } \\
\text { turen“ } \\
9 \mathrm{~b} \text { „nächstes Ziel“ } \\
15 \text { „Lernvoraussetzun- } \\
\text { gen“ } \\
17 \text { „Oliver“ }\end{array}$ & $\begin{array}{l}\text { Rechtschreiben } \\
\text { Schreiben/Grammatik } \\
\text { und DaZ } \\
\text { Schreiben/Grammatik } \\
\text { Mündlichkeit/Grammatik } \\
\text { Mündlichkeit/Grammatik } \\
\text { Schreiben/Grammatik } \\
\text { und DaZ }\end{array}$ & $\begin{array}{l}\text { geschlossen } \\
\text { geschlossen } \\
\text { geschlossen } \\
\text { geschlossen } \\
\text { geschlossen } \\
\text { geschlossen } \\
\text { geschlossen }\end{array}$ \\
\hline $\begin{array}{l}\text { FDW-Facette } \\
\text { „Pot“ (2): } \\
\text { Lernpotenzial von } \\
\text { Texten }\end{array}$ & $\begin{array}{l}\text { 2a „Knochen und } \\
\text { Gelenke“ } \\
13 \text { „IGLU“ }\end{array}$ & $\begin{array}{l}\text { Lesen } \\
\text { Lesen }\end{array}$ & $\begin{array}{l}\text { offen } \\
\text { geschlossen }\end{array}$ \\
\hline $\begin{array}{l}\text { FW: } \\
\text { Fachwissen (3) }\end{array}$ & $\begin{array}{l}6 \text { „Bildungssprache“ } \\
10 \text { „Sprachliches } \\
\text { Lernen“ } \\
12 \text { „Textelemente“ }\end{array}$ & $\begin{array}{l}\text { Linguistik } \\
\text { Sprachentwicklung } \\
\text { Linguistik }\end{array}$ & $\begin{array}{l}\text { geschlossen } \\
\text { geschlossen } \\
\text { geschlossen }\end{array}$ \\
\hline $\begin{array}{l}\text { PW: } \\
\text { Pädagogisches } \\
\text { Wissen (2) }\end{array}$ & $\begin{array}{l}5 \text { „Feedback“ } \\
11 \text { „Scaffolding“ }\end{array}$ & $\begin{array}{l}\text { Päd. Psychologie } \\
\text { Päd. Psychologie }\end{array}$ & $\begin{array}{l}\text { geschlossen } \\
\text { geschlossen }\end{array}$ \\
\hline
\end{tabular}

Die 14 genuin fachdidaktischen Items verteilen sich dabei wie folgt auf die verschiedenen Lernbereiche (siehe Tab. 1): Rechtschreiben (Item 1), Schreiben (Items 4, 7, 8, 17), Sprechen (Items 9a, 9b, 14, 15, 16) und Lesen (Items 2a, 2b, 3, 13). Items, die sich auf die Sprachproduktion beziehen, überwiegen im FaWi-S dabei deutlich, was im Hinblick auf das Ziel „sprachförderliches Professionswissen“ abzubilden, legitim ist, da Lerngelegenheiten für den aktiven Sprachgebrauch be- 
sonders zentral für den Spracherwerb sind (Gibbons, 2002). Lesen und Hören als rezeptive Sprachhandlungen sind im Test weniger ausgeprägt repräsentiert.

Die Items 6 und 12 zur Bildungssprache testen das sprachwissenschaftliche Fachwissen der Lehrkräfte. Durch die Häufung von Fachtermini ist die Lehrkraft auf sprachwissenschaftliches Grundlagenwissen angewiesen, um diese Items beantworten zu können. Ebenso testet Item 10 fachliches Grundlagenwissen im Bereich des Spracherwerbs.

Item 11 zum Scaffolding adressiert genuin pädagogisch-psychologisches Wissen. Gerade im Rahmen der Spracherwerbsforschung gilt das Scaffolding aber als eine zentrale Maßnahme für die gezielte Unterstützung sprachlich schwacher Schüler*innen (Gibbons, 2002; Quehl, 2010). Auch Item 5 zielt auf das pädagogisch-psychologische Wissen der Lehrkraft, indem verschiedene Methoden des Feedbackgebens im Kontext von schriftlichen Texten in Bezug auf ihre Effektivität eingeschätzt werden müssen (Hattie \& Timperley, 2007; Merz-Grötsch, 2011). Drei Items beziehen sich darüber hinaus explizit auf Schüler*innen mit DaZ-Hintergrund (Deutsch als Zweitsprache) und erfordern deshalb zusätzlich Wissen, das während der Lehramtsausbildung nicht im Kontext der allgemeinen Deutschdidaktik erworben wird, sondern überwiegend in den seit ca. zehn Jahren vermehrt geschaffenen DaZ-Studiengängen oder -modulen (Items 4, 7 und 17).

Bei der Entwicklung der Items wurde darauf geachtet, dass nicht nur deklaratives Wissen getestet wurde, etwa indem Fachbegriffe abgefragt werden, sondern dass es sich überwiegend um prozedurales Wissen handelt, durch das die Lehrkraft sowohl ihr explizites wie auch implizites Wissen nachweisen kann (Neuweg, 2015). So enthält ein Großteil der Items einen Schülertext oder einen Lehrtext (meist aus dem naturwissenschaftlichen Kontext). Sowohl bei den Texten der Schüler*innen als auch bei den Lehrmaterialien handelt es sich dabei durchgehend um authentische Texte, die den Lehrkräften in dieser Form auch in ihrem täglichen Unterricht begegnen. Auch die Instruktionen zielen entweder auf die diagnostische Kompetenz im Hinblick auf spezifische Schwierigkeiten von Schüler*innen, auf die Analysekompetenz im Hinblick auf Unterrichtsmaterial oder die Wahl von Unterrichtsmethoden und Hilfestellungen.

\subsection{Pilotierung des Tests}

Alle Itemvorschläge wurden von den Mitgliedern der Forschungsteams Regensburg und Landau generiert, jeweils ausführlich im Gesamtteam diskutiert und in mehreren Iterationen überarbeitet. Eine erste Testversion bestand aus 23 Items, die am ersten Messzeitpunkt (März bis Mai 2016) in zwei BiSS-Verbünden mit 80 Lehrkräften an Grundschulen (aus Sachsen-Anhalt und aus Bayern) pilotiert wurde. Aufgrund der Pilotierungsergebnisse wurden vor allem Items mit Deckeneffekten entfernt sowie einige der offen gestellten Fragen geschlossen, die schwierig zu kodieren waren (Beispiel: „Bitte nennen Sie mindestens drei Möglichkeiten, 
um die Lesekompetenz Ihrer Schülerinnen und Schüler zu überprüfen!“). Weiterhin wurden einige offensichtlich missverständliche Formulierungen verbessert. Aufgrund der Pilotierungsergebnisse ergaben sich für den FaWi-S insgesamt 19 Items mit angemessener Schwierigkeit. Für die beiden offenen Items (2a: Knochen und Gelenke, 3: Lesestrategien; siehe Tab. 1) wurde ein Kodiermanual entwickelt (die entsprechenden Interraterreliabilitäten finden sich in Tab. 4).

\section{Testvalidierung des FaWi-S}

\subsection{Stichprobe und Durchführung}

Am ersten Messzeitpunkt von Eva-Prim wurden die neukonstruierten Items des FaWi-S pilotiert und am zweiten Messzeitpunkt kam der finale Test bei insgesamt $N=86$ Grundschullehrkräften aus den Verbünden V1 bis V4 sowie aus Kontrollschulen zum Einsatz. Zur Testvalidierung wurde der FaWi-S für den vorliegenden Beitrag darüber hinaus bei $N=21$ weiteren Grundschullehrkräften und $N=346$ Grundschullehramtsstudierenden administriert, um zielgerichtet Hypothesen zu entsprechenden Statusgruppenunterschieden im Professionswissen bzw. zu dessen Zunahme während der Ausbildung belegen zu können

Der (finale) FaWi-S wurde damit bei $N=107$ Grundschullehrkräften und $N=346$ Grundschullehramtsstudierenden aus fünf Bundesländern (SachsenAnhalt, Bayern, Baden-Württemberg, Nordrhein-Westfalen und Rheinland-Pfalz) administriert. Tabelle 2 gibt einen Überblick über die Gesamtstichprobe. Vor allem bei den Studierenden handelt es sich um eine Gelegenheitsstichprobe, die zur Testvalidierung über BiSS hinaus vom Eva-Prim-Projektteam zusätzlich rekrutiert wurde. Es ist wichtig zu beachten, dass aufgrund der teilweise geringen Stichprobengrößen keine Bundeslandvergleiche intendiert sind (Tab. 2 dient hier also lediglich zur deskriptiven Übersicht über die Stichprobe). 
Tab. 2: Gesamtstichprobe (Grundschullehrkräfte und Lehramtsstudierende)

\begin{tabular}{lcccccc}
\hline & \multicolumn{3}{c}{ Lehrkräfte Grundschule } & \multicolumn{3}{c}{ Studierende Grundschule } \\
\hline Bundesland & $\boldsymbol{N}$ & $\begin{array}{c}\text { Berufserfah- } \\
\text { rung } \boldsymbol{M}(\boldsymbol{S} \boldsymbol{D})\end{array}$ & $\begin{array}{c}\% \\
\text { weiblich }\end{array}$ & $\boldsymbol{N}$ & $\begin{array}{c}\text { Fachsemester } \\
\boldsymbol{M}(\boldsymbol{S} \boldsymbol{D})\end{array}$ & $\begin{array}{c}\% \\
\text { weiblich }\end{array}$ \\
\hline $\begin{array}{l}\text { Sachsen- } \\
\text { Anhalt }\end{array}$ & 30 & $20.77(12.54)$ & 85.7 & 43 & $4.24(1.45)$ & 81.4 \\
\hline Bayern & 14 & $12.07(9.62)$ & 100 & 250 & $2.48(1.90)$ & 91.2 \\
\hline $\begin{array}{l}\text { Baden- } \\
\begin{array}{l}\text { Württem- } \\
\text { berg }\end{array}\end{array}$ & 23 & $12.75(10.26)$ & 100 & Keine Stichprobe erhoben \\
\hline $\begin{array}{l}\text { Nordrhein- } \\
\text { Westfalen }\end{array}$ & 18 & $18.82(11.61)$ & 94.1 & 35 & $8.31(1.80)$ & 97.1 \\
\hline $\begin{array}{l}\text { Rheinland- } \\
\text { Pfalz }\end{array}$ & 22 & k.A. & 100 & 19 & $5.77(2.13)$ & k.A. \\
\hline \begin{tabular}{l} 
Gesamt \\
\hline
\end{tabular} & 107 & $16.54(11.69)$ & 93.8 & 346 & $3.64(2.72)$ & 90.5 \\
\hline
\end{tabular}

$M=$ arithmetisches Mittel; $S D$ = Standardabweichung; FaWi-S = Fachdidaktisches Wissen zur Sprachförderung; k.A. = keine Angabe.

Von den $N=107$ Lehrkräften, die in die folgenden Analysen zum FaWi-S eingingen, waren 93,8 Prozent weiblich und die durchschnittliche Berufserfahrung betrug 16.5 Jahre $(S D=11.7)$. Von den $N=46$ Grundschullehramtsstudierenden waren 90.5 Prozent weiblich und die durchschnittliche Studienerfahrung betrug 3.6 Semester $(S D=2.7)$. Alle Studierenden wurden im Rahmen verschiedener universitärer Veranstaltungen unter Aufsicht einer Testleitung untersucht.

Für die Bearbeitungszeit des FaWi-S wurde ein Richtwert von 60 Minuten vorgegeben, der aber bei zusätzlichem Zeitbedarf nicht strikt eingehalten wurde. Die meisten Lehrkräfte und Studierenden blieben bei der Bearbeitung unter dem Richtwert.

\subsection{Psychometrische Gütekriterien}

Tabelle 3 gibt einen Überblick über zentrale psychometrische Gütekriterien.

\section{Interraterreliabilitat (Auswertungsobjektivität)}

Von den zwei offenen Items wurden zur Bestimmung der Auswertungsobjektivität jeweils knapp 100 Antworten der Studienteilnehmer*innen von zwei geschulten

3 Die Lehrkräfte wurden bei einer Lehrerfortbildung in Rheinland-Pfalz untersucht. Die teilnehmenden Lehrkräfte haben weder ihre Berufserfahrung noch ihr Bundesland angegeben, weshalb nicht ausgeschlossen werden kann, dass sich bei den Lehrkräften auch einige wenige Lehrkräfte aus anderen Bundesländern befanden. 
Rater*innen doppelt kodiert. Dabei konnte bei beiden Items eine hohe Übereinstimmung erzielt werden (Spearmens $r h o=.90$ bzw. .96). Das Kodiermanual für die beiden Items befindet sich ebenfalls im Online-Anhang.

\section{Interne Konsistenz des FaWi-S (Skalenreliabilität und Trennschärfen)}

Tabelle 3 informiert weiterhin über die Reliabilität der Skala FaWi-S (indiziert über Cronbachs Alpha als Maß der internen Konsistenz) sowie die part-whole korrigierten Trennschärfen $r_{i t}$ der beteiligten Items (zusätzlich sind jeweils die mittlere Trennschärfe sowie deren range abgebildet). Das intendierte Konstrukt konnte durch den FaWi-S nur eingeschränkt reliabel erfasst werden $(\alpha=.57)$. Dies ist für Testneukonstruktionen für ein solch breites Konstrukt aber durchaus akzeptabel und zudem stellt Cronbachs Alpha bei einem Tau-kongenerischen Modell - wie es hier vorliegt - nur eine Mindestschätzung der Reliabilität dar (Bühner, 2011). Die Trennschärfen der Items sind ebenfalls gerade noch akzeptabel (auch wenn es einzelne Items gibt, die die Schwelle von $r_{i t}=.20$ unterschreiten, was der Angabe „range“ zu entnehmen ist).

\section{Augenscheinvalidität (face validity)}

Alle Items des FaWi-S wurden weiterhin vier externen Expert*innen vorgelegt mit der Bitte, jeweils sowohl die Berufsrelevanz des Iteminhaltes als auch die Eindeutigkeit der Formulierung auf einer vierstufigen Skala zu beurteilen. Die Items wurden von den Expert*innen als hochgradig berufsrelevant eingestuft $(M=3,83)$. Es gab dabei kein einziges Item, das von den Expert*innen durchschnittlich schlechter als 3 bewertet wurde (in Tab. 4 sind die Mittelwerte über alle Items zur Berufsrelevanz abgebildet, die Beurteilungen der Eindeutigkeit fielen ähnlich gut aus). 
Tab. 3: Interne Konsistenz (Cronbachs $\alpha$ ) des FaWi-S, $M(S D)$ und range der Trennschärfen $\left(\mathrm{r}_{\mathrm{it}}\right)$, mittlere Augenscheinvalidität (Berufsrelevanz) über alle Items und Interraterreliabilität (Spearmans $\rho$ ) der beiden offenen Items.

\begin{tabular}{ccccc}
\hline $\begin{array}{c}\text { Skala } \\
(N=453)\end{array}$ & $\begin{array}{c}\text { Interne } \\
\text { Konsistenz }\end{array}$ & $\begin{array}{c}\text { Trennschärfe } \\
\text { (part-whole } \\
\text { korrigiert) }\end{array}$ & $\begin{array}{c}\text { Augenscheinvalidität } \\
\text { (Berufsrelevanz) }\end{array}$ & $\begin{array}{c}\text { Interrater- } \\
\text { reliabilität }\end{array}$ \\
(Itemzahl) & Cronbachs $\alpha$ & $\begin{array}{r}r_{\text {it }} \\
M(S D)\end{array}$ & $\begin{array}{c}n=4 \text { externe } \\
\text { Expert*innen } \\
M(S D)\end{array}$ & $\begin{array}{c}\text { Item 2a: } n=91 \\
\text { Item 3: } n=84 \\
\text { doppelt kodierte } \\
\text { Testhefte }\end{array}$ \\
& & & & $\begin{array}{c}\text { Spearmans } \rho \\
\text { 19 Items }\end{array}$ \\
& .57 & $\begin{array}{c}.19(.09) \\
\text { range } 0.06-0.35\end{array}$ & $\begin{array}{c}3.83(0.31) \\
\text { range 3.00-4.00 }\end{array}$ & $\begin{array}{c}\text { Item 2a: } \rho=.90 \\
\text { Item 3: } \rho=.96\end{array}$ \\
\hline
\end{tabular}

$M=$ arithm. Mittel; $S D=$ Standardabweichung; range = Spannweite; FaWi-S = Fachdidaktisches Wissen zur Sprachförderung; Skalierung Augenscheinvalidität (Berufsrelevanz) $=1$ Trifft nicht zu, 2 Trifft eher nicht zu, 3 Trifft eher zu, 4 Trifft zu.

Insgesamt kann konstatiert werden, dass die Items des FaWi-S ein breites Themenfeld abbilden, deren Inhalte (Sprachförderung im Fachunterricht) üblicherweise nicht gebündelt bzw. standardmäßig in spezifischen Veranstaltungen vermittelt werden. Die FaWi-S Skala kann deshalb zwar vor allem in der Studierendenstichprobe nur eingeschränkt als reflexives Konstrukt interpretiert werden, den Iteminhalten wird aber von externen Expert*innen durchweg eine hohe Berufsrelevanz im Hinblick auf Sprachförderung zugeschrieben (Inhaltsvalidität). Auch etliche der untersuchten Lehrkräfte attestierten den Items in persönlichen Gesprächen Relevanz für die unterrichtliche Praxis. Vergleichsweise geringe Reliabilitäten sind gerade bei Test(neu)konstruktionen zum Professionswissen durchaus üblich (z.B. Krauss et al., 2017) und können gerade bei breiten Konstrukten zugunsten der Inhaltsvalidität eingeschränkt sein (Bühner, 2011).

\subsection{Deskriptive Ergebnisse, Statusgruppenunterschiede und Ausbildungssensitivität}

In Tabelle 4 (unten) finden sich der von der Gesamtstichprobe erreichte Mittelwert (und die Standardabweichung) sowie das empirische Maximum und Minimum für den FaWi-S. Weiterhin werden die Unterschiede zwischen Lehrkräften und Studierenden berichtet. 
Tab. 4: Mittelwerte $(M)$, Standardabweichungen $(S D)$ und empirische Minima und Maxima des FaWi-S für die Gesamtstichprobe sowie getrennt für die einzelnen Bundesländer. In der letzten Spalte werden die $p$-Werte aus $\mathrm{t}$-Tests und die Effektstärken $d$ für den Gruppenvergleich zwischen Lehrkräften und Studierenden angegeben.

\begin{tabular}{lcccccc}
\hline FaWi-S & \multicolumn{2}{c}{ Lehrkräfte und Studierende } & Lehrkräfte & Studierende & $\begin{array}{c}\text { Gruppen- } \\
\text { vergleich }\end{array}$ \\
\cline { 2 - 7 } & $M(S D)$ & Emp. & Emp. & $M(S D)$ & $M(S D)$ & $p / d$ \\
& $N$ & Min & Max & $N$ & $N$ & \\
\hline Sachsen- & $16.96(3.91)$ & 6.64 & 24.37 & $19.23(3.18)$ & $15.37(3.69)$ & $p<.001$ \\
Anhalt & 73 & & & 30 & 43 & $d=1.11$ \\
\hline Bayern & $18.69(2.59)$ & 12.15 & 26.75 & $21.38(2.93)$ & $18.53(2.39)$ & $\begin{array}{c}\mathrm{p}<.001 \\
\end{array}$ \\
& 264 & & & 14 & 250 & $d=1.18$ \\
\hline BW & $20.59(2.71)$ & 16.39 & 27.53 & $20.59(2.71)$ & nicht & - \\
& 23 & & & 23 & erhoben & \\
\hline NRW & $18.35(3.04)$ & 9.08 & 24.68 & $18.09(4.22)$ & $18.49(2.28)$ & $p=.71$ \\
& 53 & & & 18 & 35 & $d=-0.13$ \\
\hline Rheinland- & $19.26(3.24)$ & 8.83 & 25.62 & $20.16(3.67)$ & $18.17(2.27)$ & $p=.05$ \\
Pfalz & 40 & & & 22 & 18 & $d=0.64$ \\
\hline Gesamt & $18.51(3.01)$ & 6.64 & 27.53 & $19.80(3.45)$ & $18.11(2.75)$ & $\mathrm{p}<.001$ \\
& 453 & & & 107 & 346 & $d=0.58$ \\
\hline
\end{tabular}

$M=$ arithmetisches Mittel; $S D=$ Standardabweichung; FaWi-S = Fachdidaktisches Wissen zur Sprachförderung. Die Effektstärke $d$ berechnet sich aus der Mittelwertdifferenz beider Gruppen $\left(\mathrm{M}_{\text {Lehrer }}-\right.$ $\mathrm{M}_{\text {Studierende }}$ ) dividiert durch die gepoolte Standardabweichung.

Nach Cohen (1992) entspricht eine Effektstärke von $d=.20$ einem kleinen, von $d=.50$ einem mittleren und von $d=.80$ einem großen Effekt.

Darüber hinaus sind in Tabelle 4 (oben) bundesland- und statusgruppenspezifische Mittelwerte angegeben. Vergleiche zwischen Bundesländern (und somit auch zwischen BiSS-Verbünden) sollen aufgrund der geringen Fallzahlen hier allerdings unterbleiben (Rank et al., im Druck). Die bundeslandspezifischen Statusgruppenvergleiche in Tabelle 4 sind jedoch zur Erhöhung der Aussagekraft der Ergebnisse erforderlich, da es bei bildungsbezogenen Kompetenzmaßen in der Regel starke Unterschiede zwischen Bundesländern gibt (dies gilt nicht nur für Schülerleistungen wie z.B. bei PISA, sondern auch in Bezug auf professionelle Lehrerkompetenzen, z.B. Krauss et al., 2017) und beim Gesamtvergleich (unten) nicht nach der jeweiligen Stichprobengröße gewichtet wurde.

Abgesehen von NRW zeigen sich hier jeweils große Statusgruppenunterschiede zugunsten der Lehrkräfte, was ein deutlicher Indikator dafür ist, dass der FaWi$S$ Professionswissen im eigentlichen Sinne erfasst, da die der Profession bereits angehörigen Studienteilnehmer*innen über signifikant größere Wissensbestände 
verfügen. Die besseren Ergebnisse der Studierenden in NRW können darauf zurückgeführt werden, dass die Tests in einem Seminar durchgeführt wurden, das Wissen aus dem Inhaltsbereich des Tests vermittelte.

Als weiteren Hinweis auf die Ausbildungssensitivität des Tests können einzelne positive und teilweise signifikante Korrelationen des FaWi-S mit der Semesterzahl in den Studierendengruppen gesehen werden (Tab. 5). Auch wenn sie in den verschiedenen Bundesländern durchaus differenziell ausfallen (und auch aufgrund der geringen Stichprobengrößen nicht überbewertet werden sollten), indizieren sie insgesamt eine Wissenszunahme während der universitären Ausbildungsphase. Die fehlenden (oder teilweise sogar negativen) Korrelationen mit der Berufserfahrung in der Lehrergruppe sind für Professionswissenstests nicht unüblich (Blömeke et al., 2011; Kirschner et al., 2017; Krauss et al., 2017) und im vorliegenden Fall sicher auch darauf zurückzuführen, dass das Thema "Sprache im Fach" erst seit etwa zehn Jahren intensiver untersucht wird und deshalb kaum zu einem traditionellen Ausbildungsthema im Lehramtsstudium gezählt werden kann. Über Ausbildungswissen zu den Themen "Sprachförderung" und "Sprache im Fach“ verfügen deshalb in der Regel nur jüngere Lehrkräfte.

Tab. 5: Korrelationen $r$ (Pearson) zwischen FaWi-S und der Semesterzahl (Studierende) bzw. der Berufserfahrung in Jahren (Lehrkräfte).

\begin{tabular}{lcc}
\hline \multicolumn{1}{c}{ FaWi-S } & $\begin{array}{c}\text { Grundschulstudierende } \\
\text { Korrelation mit } \\
\text { Semester }\end{array}$ & $\begin{array}{c}\text { Grundschullehrkräfte } \\
\text { Korrelation mit Berufserfahrung } \\
\text { (in Jahren) }\end{array}$ \\
\hline Sachsen-Anhalt & .10 & $-.43^{*}$ \\
\hline Bayern & $.23^{* *}$ & -.17 \\
\hline $\begin{array}{l}\text { Baden- } \\
\text { Württemberg }\end{array}$ & + & -.35 \\
\hline NRW & .17 & .08 \\
\hline Rheinland-Pfalz & $.68^{*}$ & ++ \\
\hline
\end{tabular}

+: In Baden-Württemberg wurden keine Daten mit Grundschulstudierenden erhoben.

++: Bei den Lehrkräften aus Rheinland-Pfalz wurde die Berufserfahrung nicht erhoben.

\subsection{Konfirmatorische Faktorenanalyse}

Abschließend wurde mit Hilfe einer latenten konfirmatorischen Faktorenanalyse die Eindimensionalität des FaWi-S auf der Grundlage aller Studienteilnehmer*innen $(N=510)$ überprüft (Abb. 2). Aufgrund der relativ großen Itemzahl wurden zur Überidentifizierung des Modells aus den 19 Items vier Zufallsparcels gebildet, so dass die Gesamtvarianz pro Parcel in etwa vergleichbar ist (Bühner, 2011). Abbildung 2 verdeutlicht den hervorragenden Fit des Modells, was als ein weiterer 
Hinweis auf die Validität des latenten Konstrukts „Sprachförderwissen“ gesehen werden kann.

Die Fit-Indizes in Abbildung 2 verdeutlichen dabei, dass es trotz der breiten Konzeptualisierung (Items zum fachdidaktischen, aber auch zum pädagogischen Wissen und zum Fachwissen) gelungen ist, die Skala des FaWi-S als eindimensionales Konstrukt anzulegen. Somit eignet sich der Test auch trotz eingeschränkter Reliabilität gut zur Erhebung des sprachförderlichen Wissens von Grundschullehrkräften.

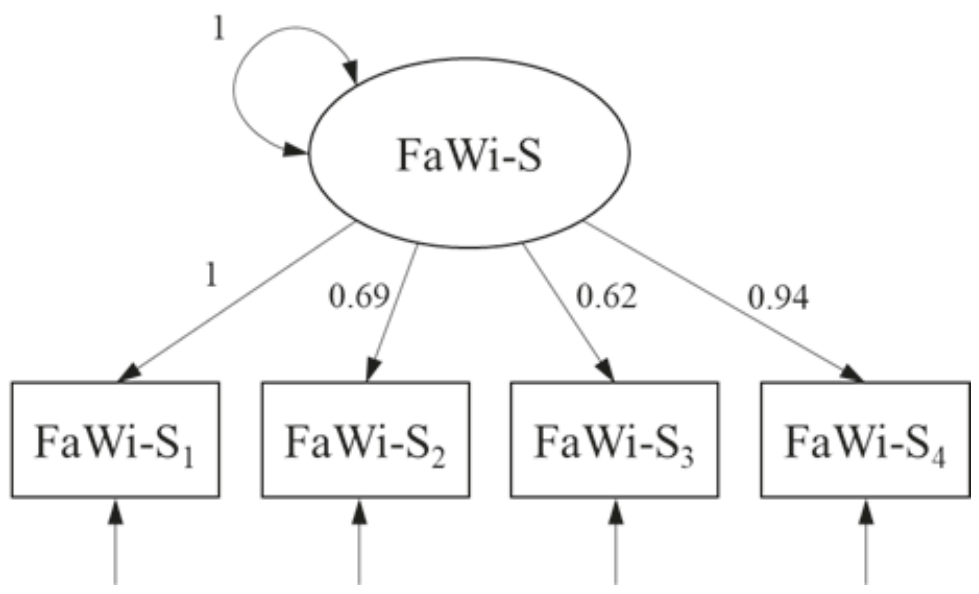

Modellfit: $\chi^{2}(2, N=510 ; p=.17)=3.55 ; C F I=1 ; R M S E A=.04 ; S R M R=.01$.

Werte von $x^{2} \leq 3 \mathrm{df}, p \geq .01$, CFI (Comparative Fit Index) $\geq .95$, RMSEA (Root-Mean-Square Error of Approximation) $\leq .08$ und $S R M R$ (Standardized Root Mean Residual) $\leq .10$ indizieren akzeptablen Modellfit (Hu \& Bentler, 1999; Schermelleh-Engel \& Moosbrugger \& Müller, 2003).

Abb. 2: Konfirmatorisches Faktorenmodell des fachdidaktischen Wissens zur Sprachförderung (latente Modellierung auf Basis der Gesamtstichprobe $N=510$ ).

\section{Fazit}

Der FaWi-S bildet das Sprachförderwissen von Grundschullehrkräften auf einer mittleren Granularitätsebene ab, ist also weniger umfassend als FALKO (Krauss et al., 2017) oder TEDS-LT (Blömeke et al., 2011), aber auch nicht so spezifisch auf einen Bereich zugeschnitten wie etwa der Professionstest zum Rechtschreiben von Riegler und Wiprächtinger-Geppert (2016). Mit dem FaWi-S liegt damit ein Instrument vor, das für weitere Forschungsprojekte im Bereich des Themas "Sprache im Fach" genutzt werden kann. Im Rahmen von Eva-Prim werden zentrale und zukünftig noch zu beantwortende Fragestellungen sein, ob sich zum 
einen durch die Teilnahme an BiSS Veränderungen im sprachbezogenen fachdidaktischen Wissen bei Lehrkräften ergeben und ob zum anderen das im FaWi-S erhobene Sprachförderwissen prädiktiv valide für die Unterrichtsqualität und den Lernzuwachs der Schüler*innen ist.

Der FaWi-S ist psychometrisch gut geeignet, um diese Fragestellungen in einem nächsten Schritt unter Einbeziehung der Daten aus dem dritten Messzeitpunkt anzugehen. Die bislang vorliegenden Daten deuten darauf hin, dass die Teilnahme an BiSS nicht zu einem substanziellen Zuwachs an Sprachförderwissen geführt hat. Auch wenn sich dieses erste Ergebnis in genaueren Analysen bestätigt, ist dies zwar zunächst enttäuschend, aber im Hinblick auf die Entwicklung ähnlicher Initiativen bedeutsam, zeigt es doch, dass der Erwerb von Sprachförderwissen auf substanziellere Maßnahmen angewiesen ist als die in BiSS vorgesehenen Fortbildungen.

Der FaWi-S lässt sich in Zukunft auch einsetzen, um weitere Fragestellungen, die sich im Bereich der Sprachförderung stellen, zu untersuchen. So könnte er genutzt werden, um Nachqualifizierungsprogramme im Bereich der Sprachförderung zu evaluieren oder um Unterschiede zwischen Populationen bzw. Entwicklungen dieser zu messen. Von besonderem Interesse dürfte es sein, auch im Rahmen von anderen Projekten zu untersuchen, wie sich ein höheres Sprachförderwissen von Lehrkräften auf die sprachlichen und fachlichen Kompetenzen von Kindern auswirkt. Beachtet werden muss allerdings, dass sich die Textvignetten auf Beispiele aus der Primarstufe beziehen. Nur Grundschullehrkräfte können deshalb Erfahrungswissen heranziehen, um einen Großteil der Items zu beantworten.

\section{Literatur}

Becker-Mrotzek, M., Schramm, K, Thürmann, E. \& Vollmer, H. J. (Hrsg.) (2013). Sprache im Fach. Münster: Waxmann.

Blömeke, S., Bremerich-Vos, A., Haudeck, H., Kaiser, G., Nold, G., Schwippert, K. \& Willenberg, H. (Hrsg.) (2011). Kompetenzen von Lehramtsstudierenden in gering strukturierten Domänen, Erste Ergebnisse aus TEDS-LT. Münster: Waxmann.

Blömeke, S., Kaiser, G. \& Lehmann, R. (Hrsg.) (2010). TEDS-M 2008. Professionelle Kompetenz und Lerngelegenheiten angehender Primarstufenlehrkräfte im internationalen Vergleich. Münster: Waxmann.

Bochnik, K. \& Ufer, S. (2016). Die Rolle (fach-)sprachlicher Kompetenzen zur Erklärung mathematischer Kompetenzunterschiede zwischen Kindern mit deutscher und nicht-deutscher Familiensprache. Zeitschrift für Grundschulforschung 9(1), 135-147.

Bredel, U. (2007). Sprachbetrachtung und Grammatikunterricht. Paderborn: UTB.

Bos, W., Wendt, H., Ünlü, A., Valtin, R., Euen, B., Kasper, D. \& Tarelli, I. (2012). Leistungsprofile von Viertklässlerinnen und Viertklässlern in Deutschland. In W. Bos, I. Tarelli, A. Bremerich-Vos \& K. Schwippert (Hrsg.), IGLU 2011. Lesekompetenzen von Grundschulkindern in Deutschland im internationalen Vergleich (S. 227-259). Münster: Waxmann.

Bühner, M. (2011). Einführung in die Test-und Fragebogenkonstruktion. Pearson Deutschland GmbH. Cohen, J. (1992). A power primer. Psychological bulletin, 112(1), 155.

Feilke, H. \& Tophinke, D. (2016). Grammatisches Lernen. Praxis Deutsch 43, 256, 4-11. 
Gaier, L. \& Schilcher, A. (in Vorb.). Gutes Erklären im Deutschunterricht - eine empirische Studie zu unterschiedlichen Beobachterperspektiven. In A. Schilcher, S. Krauss, A. Lindl \& S. Hilbert (Hrsg.), FALKE - Fachspezifische Lehrer*innenkompetenzen im Erklären. Ein interdisziplinäres Forschungsprojekt. Weinheim: Beltz.

Gibbons, P. (2002). Scaffolding language, scaffolding learning. Teaching second language learners in the mainstream classroom. Portsmouth NH: Heinemann.

Gogolin, I. (2008). Herausforderung Bildungssprache. Die Grundschulzeitschrift, 23(215/216), 26.

Goldenstein, M. (in Vorb.). Förderung der schriftlichen Erzählkompetenz von Grundschülern der 4. Jahrgangsstufe durch ein selbstreguliertes Lese- und Schreibtraining mit speziellem Fokus auf die textuellen Veränderungen bei leistungsstarken Kindern. Dissertation, Universität Regensburg.

Hagena, M., Leiss, D., Neumann, A. \& Schwippert, K. (2015). Durch Sprachförderung zum fachlichen Erfolg? In F. Caluori, H. Linneweber-Lammerskitten \& C. Streit (Hrsg.), Beiträge zum Mathematikunterricht 2015 (S. 348-351). Münster: WTM.

Hattie, J. \& Timperley, H. (2007). The Power of Feedback. Review of Educational Research, 77(1), 81-112.

Hill, H. C., Rowan, B. \& Ball, D. (2005). Effects of teachers' mathematical knowledge for teaching on student achievement. American Educational Research Journal 42, 371-406.

Hu, L. T. \& Bentler, P. M. (1999). Cutoff criteria for fit indexes in covariance structure analysis: Conventional criteria versus new alternatives. Structural equation modeling: a multidisciplinary journal, 6(1), 1-55.

Kirschner, S., Sczudlek, M., Tepner, O., Borowski, A., Fischer, H. E., Lenske, G., Leutner, D., Neuhaus, B. J., Sumfleth, E., Thillmann, H. \& Wirth, J. (2017). Professionswissen in den Naturwissenschaften (ProwiN). In C. Gräsel \& K. Trempler (Hrsg.), Entwicklung von Professionalität pädagogischen Personals. Interdisziplinäre Betrachtungen, Befunde und Perspektiven (S. 113-130). Wiesbaden: VS.

KMK (2004). Sekretariat der Ständigen Konferenz der Kultusminister der Länder in der Bundesrepublik Deutschland (2004). Bildungsstandards im Fach Deutsch für den Primarbereich. Neuwied: Luchterhand.

König, J., Doll, J., Buchholtz, N., Förster, S., Kaspar, K., Rühl, A.-M., Strauß, S., Bremerich-Vos, A., Fladung, I. \& Kaiser, G. (2017). Pädagogisches Wissen versus fachdidaktisches Wissen? Struktur des professionellen Wissens bei angehenden Deutsch-, Englisch- und Mathematiklehrkräften im Studium. Zeitschrift für Erziehungswissenschaft, 1-28.

Krauss, S., Lindl, A., Schilcher, A. \& Tepner O. (2017). Das Forschungsprojekt FALKO - ein einleitender Überblick. In S. Krauss, A. Lindl, A. Schilcher, M. Fricke, A. Göhring, B. Hofmann, P. Kirchhoff \& R. H. Mulder (Hrsg.), FALKO: Fachspezifische Lehrerkompetenzen. Konzeption von Professionswissenstests in den Fächern Deutsch, Englisch, Latein, Physik, Musik, Evangelische Religion und Pädagogik (S. 9-65). Münster: Waxmann.

Krauss, S. \& Bruckmaier, G. (2014). Das Experten-Paradigma in der Forschung zum Lehrerberuf. In E. Terhart, H. Bennewitz \& M. Rothland (Hrsg.), Handbuch der Forschung zum Lehrberuf, (2. Aufl., S. 241-261). Münster: Waxmann.

Kunter, M., Baumert, J., Blum, W., Klusmann, U., Krauss, S. \& Neubrand, M. (Hrsg.) (2011). Professionelle Kompetenz von Lehrkräften, Ergebnisse des Forschungsprogramms COACTIV. Münster: Waxmann.

Kunter, M., Klusmann, U., Baumert, J., Richter, D., Voss, T. \& Hachfeld, A. (2013). Professional competence of teachers: effects on instructional quality and student development. Journal of Educational Psychology, 105(3), 805-820.

Lenske, G., Wagner, W., Wirth, J., Thillmann, H., Cauet, E., Liepertz, S. \& Leutner, D. (2016). Die Bedeutung des pädagogisch-psychologischen Wissens für die Qualität der Klassenführung und den 
Lernzuwachs der Schüler/innen im Physikunterricht. Zeitschrift für Erziehungswissenschaft, 19(1), 211-233.

Leutner, D., Klieme, E., Meyer, K. \& Wirth, J. (2004). Problemlösen. In M. Prenzel, J. Baumert, W. Blum, R. Lehmann, D. Leutner, M. Neubrand, R. Pekrun, H.-G. Rolff, J. Rost \& U. Schiefele (Hrsg.), PISA 2003. Der Bildungsstand der Jugendlichen in Deutschland? Ergebnisse des zweiten internationalen Vergleichs (S. 147-175). Münster: Waxmann.

Merkert, A. (in Vorb.). SAMT: Sprachliche Ausdrucksfähigkeit in Mathematik - eine Ratingskala zur Messung der schriftsprachlichen Kompetenzen von Dritt-und Viertklässlern. Dissertation, Universität Landau.

Merz-Grötsch, J. (2011). Gutes Feedback geben. Probleme diagnostizieren - Schreibkompetenzen fördern. Grundschule, 3, 30-33.

Müller-Hill, E. (2016). Warum „immer“ so - und nicht anders? Erklären (-warum) im Mathematikunterricht mittels operativer Invarianz entlang kontrastiver Leitfragen. In Institut für Mathematik und Informatik Heidelberg (Hrsg.), Beiträge zum Mathematikunterricht 2016 (S. 1119-1122). Münster: WTM.

Neuweg, H. G. (2015). Kontextualisierte Kompetenzmessung. Zeitschrift für Pädagogik, 3, 377-383.

Papadopoulou, P. \& Bescherer, C. (2016). Einsicht in das Sprachhandeln angehender Mathematiklehrkräfte mit Hilfe von Podcasts. In Institut für Mathematik und Informatik Heidelberg (Hrsg.), Beiträge zum Mathematikunterricht 2016 (S.731-734). Münster: WTM.

Pissarek, M. \& Schilcher, A. (2017): FALKO-D. Die Untersuchung des Professionswissens von Deutschlehrenden. Entwicklung eines Messinstruments zur fachspezifischen Lehrerkompetenz und Ergebnisse zu dessen Validierung. In S. Krauss, A. Lindl, A. Schilcher, M. Fricke, A. Göhring, B. Hofmann, P. Kirchhoff \& R. H. Mulder (Hrsg.), FALKO: Fachspezifische Lehrerkompetenzen. Konzeption von Professionswissenstests in den Fächern Deutsch, Englisch, Latein, Physik, Musik, Evangelische Religion und Pädagogik (S. 67-112). Münster: Waxmann.

Prediger, S. (2013). Darstellungen, Register und mentale Konstruktion von Bedeutungen und Beziehungen. Mathematikspezifische sprachliche Herausforderungen identifizieren und bearbeiten. In M. Becker-Mrotzek, K. Schramm, E. Thürmann \& H.-J. Vollmer (Hrsg.), Sprache im Fach (S. 167-183). Münster: Waxmann.

Prediger, S., Renk, N., Büchter, A., Gürsoy, E. \& Benholz, C. (2013). Family back-ground or language disadvantages? Factors for underachievement in high stakes tests. In A. Lindmeier \& A. Heinze (Eds.), Proceedings of the 37th Conference of the International Group for the Psychology of Mathematics Education, Vol. 4 (pp 4.49-4.59). Kiel: PME.

Prediger, S., Wilhelm, N., Büchter, A., Benholz, C. \& Gürsoy, E. (2015). Sprachkompetenz und Mathematikleistung - Empirische Untersuchung sprachlich bedingter Hürden in den Zentralen Prüfungen, Journal für Mathematik-Didaktik, 36(1), 77-104.

Prediger, S. (2015). Sprachförderung im Mathematikunterricht - Ein Überblick zu vernetzten Entwicklungsforschungsstudien. In F. Caluori, H. Linneweber-Lammerskitten \& C. Streit (Hrsg.), Beiträge zum Mathematikunterricht 2015 (S. 720-723). Münster: WTM.

Quehl, T. (2010). Die Möglichkeiten des Scaffolding. Zur Gestaltung des Übergangs von der Alltagssprache der Kinder zur Fach- und Bildungssprache. Grundschulunterricht Deutsch (4), 28-32.

Rank, A., Deml, I., Lenske, G., Merkert, A., Binder, K., Schulte, M., Schilcher, A., Wildemann, A., Bien-Miller, L. \& Krauss, S. (im Druck). Eva-Prim: Evaluation von Sprachförderkompetenz und (bildungs)sprachlichen Leistungen von Schülerinnen und Schülern in Mathematik. In S. Gentrup, S. Henschel, K. Schotte, L. Beck \& P. Stanat (Hrsg.). Sprach- und Schriftsprachforderung gestalten: Evaluation von Qualität und Wirksamkeit umgesetzter Konzepte. Stuttgart: Kohlhammer.

Riegler, S. \& Wiprächtiger-Geppert, M. (2016). Konzeptneutral und unterrichtsnah. Ein Instrument zur Erfassung des Professionswissens zu Orthographie und Orthographieerwerb. In H. Zimmer- 
mann \& A. Peyer (Hrsg.), Wissen und Normen. Facetten professioneller Kompetenz von Lehrkräften (S. 199-219). Frankfurt: Peter Lang.

Schermelleh-Engel, K., Moosbrugger, H. \& Müller, H. (2003). Evaluating the fit of structural equation models: Tests of significance and descriptive goodness-of-fit measures. Methods of psychological research online, 8(2), 23-74.

Schilcher, A., Röhrl, S. \& Krauss, S. (2017). Mathematik und Sprache - Sprache und Mathematik? Befunde und Herausforderungen empirisch fachdidaktischer Forschung. In D. Leiss, M. Hagena, A. Neuman \& K. Schwippert (Hrsg.), Mathematik und Sprache. Empirischer Forschungsstand und unterrichtliche Herausforderungen (S. 11-42). Münster: Waxmann.

Schilcher, A., Krauss, S., Lindl, A. \& Hilbert, S. (in Vorb.). Erklären im interdisziplinären Diskurs das Projekt FALKE. In A. Schilcher, S. Krauss, A. Lindl \& S. Hilbert (Hrsg.), FALKE - Fachspezifische Lehrer*innenkompetenz Erklären. Ein interdisziplinäres Forschungsprojekt. Münster: Waxmann.

Schulte, M. (in Vorb.). Wie fördern Lehrkräfte Bildungssprache im Fachunterricht? - Erfassung und Auswirkungen von Sprachverhalten und sprachdidaktischem Handeln im Rahmen der Studie "Eva-Prim". Dissertation, Universität Regensburg.

Shulman, L. S. (1986). Those who understand: knowledge growth in teaching. Educational Researcher, 15(2), 4-14.

Shulman, L. S. (1987). Knowledge and teaching: foundations of the new reform. Harvard Educational Review, 57(1), 1-22.

Südkamp, A. \& Praetorius, A.-K. (2017). Diagnostische Kompetenz von Lehrkräften. Münster: Waxmann.

Ufer, S., Reiss, K. \& Mehringer, V. (2013). Sprachstand, soziale Herkunft und Bilingualität: Effekte auf Facetten mathematischer Kompetenz. In M. Becker-Mrotzek, K. Schramm, E. Thürmann \& H.-J. Vollmer (Hrsg.), Sprache im Fach (S. 185-291). Münster: Waxmann.

Voss, T., Kunina-Habenicht, O., Hoehne, V. \& Kunter, M. (2015). Stichwort Pädagogisches Wissen von Lehrkräften: Empirische Zugänge und Befunde. Zeitschrift für Erziehungswissenschaft, 18(2), 187-223.

Wild, J. \& Schilcher, A. (2019). Das Regensburger Analysetool für Texte - RATTE. In A. Wehner \& F. Kirchner (Hrsg.), Sprachschätze. Materialsammlung (1-4). Halle (Salle): o. V.

Zlatkin-Troitschanskaia, O., Beck, K., Sembill, D., Nickolaus, R. \& Mulder, R. (2009). Lehrerprofessionalität. Bedingungen, Genese, Wirkungen und ihre Messung. Weinheim: Beltz.

\section{Autor*innen}

Prof. Dr. Anita Schilcher

Universität Regensburg/Lehrstuhl für Didaktik der deutschen Sprache und Literatur

Universitätsstr. 31, 93053 Regensburg

anita.schilcher@ur.de

Dr. Karin Binder

Universität Regensburg/Lehrstuhl für Didaktik der Mathematik

Universitätsstr. 31, 93053 Regensburg

karin.binder@ur.de

Prof. Dr. Stefan Krauss

Universität Regensburg/Lehrstuhl für Didaktik der Mathematik 
Universitätsstr. 31, 93053 Regensburg

stefan.krauss@ur.de

Magdalena Schulte

Ernst-Mach-Gymnasium

Jagdfeldring 82, 85540 Haar

mschulte@emg-haar.de

Prof. Dr. Astrid Rank

Universität Regensburg/Lehrstuhl für Grundschulpädagogik

Universitätsstr. 31, 93053 Regensburg

astrid.rank@ur.de

Dr. Isabell Deml

Universität Regensburg/Lehrstuhl für Grundschulpädagogik

Universitätsstr. 31, 93053 Regensburg

isabell.deml@ur.de

Prof. Dr. Sven Hilbert

Universität Regensburg/Methoden der empirischen Bildungsforschung

Universitätsstr. 31, 93053 Regensburg

sven.hilbert@ur.de

Dieser Artikel ist inklusive Anhang abrufbar unter:

https://doi.org/10.35468/5801_03 


\section{Online Anhang}

Dieses Herausgeberwerk und alle vorgestellten Instrumente zur Erfassung institutioneller (schrift-)sprachlicher Bildung sind online verfügbar unter: https://doi.org/10.35468/5801 


\section{FaWi-S \\ Testinstrument zur Erfassung des sprachförderlichen Wissens von Grundschullehrkräften}

Magdalena Schulte, Alexandra Merkert, Anita Schilcher, Lena Bien-Miller, Gerlinde Lenske, Anja Wildemann, Karin Binder, Astrid Rank \& Isabell Deml

Zitiervorschlag:

Schulte, M., Merkert, A., Schilcher, A., Bien-Miller, L., Lenske, G., Wildemann, A., Binder, K., Rank, A. \& Deml, I. (2019). FaWi-S: Testinstrument zur Erfassung des sprachförderlichen Wissens von Grundschullehrkräften. In K. Mackowiak, C. Beckerle, S. Gentrup \& C. Titz (Hrsg.), Instrumente zur Erfassung institutioneller (schrift-)sprachlicher Bildung (Online-Anhang). Bad Heilbrunn: Klinkhardt. Verfügbar unter: https://doi.org/10.35468/5801_03 
Inhalt

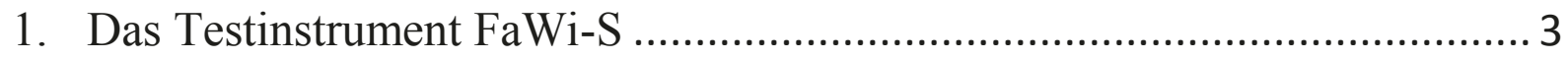

2. Kodiermanual für quantitative Aufgaben ................................................ 16

3. Kodiermanual für qualitative Aufgaben .................................................. 31

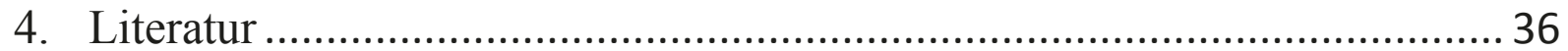


1. Das Testinstrument FaWi-S 


\begin{tabular}{|c|c|c|c|}
\hline 1. & \multicolumn{3}{|c|}{$\begin{array}{l}\text { Welche orthographischen Regeln beherrscht Tim bereits und welche noch nicht? } \\
\text { Kreuzen Sie an! }\end{array}$} \\
\hline & \multicolumn{3}{|c|}{$\begin{array}{l}\text { Tim berichtet in einer Hausaufgabe von seiner großen Leidenschaft: } \\
\text { „Mein Fusball klub } \\
\text { Fusball finde ich gut. Am 24. hbe isch ein Freundschafts Spil. Ich bin ser schnell und hbe } \\
\text { oft den Ball. Auf den Ballen stet soga der Name von unserm Ferein. Plötzlich kam ein } \\
\text { bessera Fusball Spila auf den Plats und schist ein Tor. Auf dem Plaz trenirn wir imma. } \\
\text { Wir schisen noch zwei Tore und hbn die andern besigt. Dann gab es Pizza für alle!“ }\end{array}$} \\
\hline & & $\begin{array}{l}\text { wird noch nicht } \\
\text { beherrscht }\end{array}$ & wird beherrscht \\
\hline a) & Verdoppelung der Konsonanten & 0 & O \\
\hline b) & $\begin{array}{l}\text { phonologisches Prinzip } \\
\text { (Lautgetreue Schreibweise) }\end{array}$ & O & O \\
\hline c) & $\begin{array}{l}\text { Silbisches Prinzip } \\
\text { (Berücksichtigung der Silbenstruktur) }\end{array}$ & O & O \\
\hline d) & Vokaldehnung und -kürzung & O & O \\
\hline e) & Groß- und Kleinschreibung & O & O \\
\hline f) & Bildung von Komposita & O & O \\
\hline g) & Flexion schwacher Verben & 0 & 0 \\
\hline
\end{tabular}




\section{Bitte lesen Sie den folgenden Sachtext, der für eine dritte Klasse bestimmt ist!}

\section{Knochen und Gelenke}

Die Knochen schützen und stützen den Körper. Ein Knochen besteht aus vielen tausend Knochenzellen, die wie alle Zellen von Blutgefäßen mit Sauerstoff und Nährsalzen versorgt werden. Umgekehrt geben sie aber auch Abfallstoffe ins Blut ab.

Die dünne äußere Hülle nennt man Knochenhaut. Sie erfüllt eine wichtige Funktion beim Wachstum und der Erneuerung (Regeneration) der Knochen. Im Inneren der großen Knochen befindet sich das Knochenmark. Dort werden die roten und weißen Blutzellen gebildet. Am Skelett setzen außerdem auch die Muskeln an.

Gelenke und Muskeln ermöglichen die Bewegung des Körpers. In einem Gelenk treffen zwei oder mehrere Knochen zusammen. Es gibt verschiedene Arten von Gelenken. Das Scharniergelenk sorgt zum Beispiel dafür, dass wir unser Knie beim Hinsetzen beugen können.

Jeder Mensch, also auch du, besitzt über 100 Gelenke und etwa 206 Knochen. Der kleinste Knochen ist der Steigbügel im Ohr und der größte ist der Oberschenkelknochen.

2a) Welche Merkmale des Textes können das Leseverstehen erschweren? Identifizieren Sie mögliche sprachliche Barrieren!

2b) Welche Hilfen würden Sie für Schülerinnen und Schüler anbieten, die schon häufig Probleme beim Leseverstehen zeigten?

Sie können sich für eine oder mehrere Antworten entscheiden.

a) Ich stelle Informationstexte zur Verfügung, welche die verwendeten Fachbegriffe erläutern. Dazu können z.B. Lexika oder Sachbücher genutzt werden.

b) Ich ergänze den Text um anatomische Abbildungen, die den Inhalt graphisch darstellen. Die Kombination von Text und Bild kann auf diese Weise das Verstehen unterstützen.

c) Ich streiche die meisten Fachbegriffe und kürze den Text in seiner Länge, um den Schwierigkeitsgrad zu reduzieren.

Ich markiere Schlüsselwörter, vergrößere die Schrift sowie den Zeilenabstand und unterstreiche

d) die wichtigsten Informationen, um die Identifikation von zentralen Wörtern und Sätzen zu erleichtern. 
3. Nennen Sie Lesestrategien für den weiterführenden Leseunterricht (nicht: Leselernmethoden)!

4. Dhakiya ist vor einem Jahr nach Deutschland gekommen. Nun besucht sie die dritte Klasse und schreibt über ihren neunten Geburtstag, den sie vor zwei Wochen feierte.

Wie können Sie die Schülerin ausgehend von diesem Text fördern?

Sie können sich für eine oder mehrere Antworten entscheiden.

\begin{tabular}{|c|c|}
\hline & $\begin{array}{l}\text { „Mein Geburtstag } \\
\text { Morgens gab es viele Pakete. In den Paketen waren viel schöne Sachen. In der Schule die } \\
\text { anderen Kinder sangen ein Lid für mich. Das freute mich! } \\
\text { Nachmittags andere Kinder kamen zu mir nach Hause. Meine Mutter backte einen } \\
\text { schönen Kuchen. Die Kinder und ich spielten viel. Das war Luchsus! } \\
\text { Abends ging ich schlafen und der Tag war vorbei. Das war schade! Ich möchte haben } \\
\text { jeden Tag Geburtstag!“ }\end{array}$ \\
\hline a) & $\begin{array}{l}\text { Ich kennzeichne Rechtschreib- und Grammatikfehler sowie Wortwiederholungen, damit die } \\
\text { Schülerin diese selbstständig mit einem Wörterbuch verbessern kann. }\end{array}$ \\
\hline b) & $\begin{array}{l}\text { Ich schreibe der Schülerin unter ihren Text, was sie bereits gut kann und bekräftige sie auf } \\
\text { diese Weise in ihren Bemühungen. Die Fehler lasse ich unberücksichtigt, um O } \\
\text { Frustrationserlebnisse zu vermeiden. }\end{array}$ \\
\hline c) & $\begin{array}{l}\text { Ich biete der Schülerin spezielle Grammatikübungen zur Verbstellung im Deutschen an, } \\
\text { damit sie ihre grammatischen Kenntnisse vertiefen kann. Im Anschluss daran bitte ich sie, } \\
\text { ihren Text erneut durchzusehen. }\end{array}$ \\
\hline d) & $\begin{array}{l}\text { Ich bitte im Sinne des „Peer-Tutoring“ einen besonders leistungsstarken Mitschüler bzw. } \\
\text { eine Mitschülerin den Text zu korrigieren und ziehe mich zurück. Kinder finden meist einen } \\
\text { besseren Zugang zueinander, was sich positiv auf den Lernprozess auswirkt. }\end{array}$ \\
\hline
\end{tabular}


5. $\quad$ Feedback ist bei schriftlichen Texten für ein Kind dann besonders förderlich, wenn... Sie können sich für genau eine Antwort entscheiden.

a) sich insbesondere darauf konzentriert wird, was dem Kind schon gut gelungen ist. Um eine Defizitorientierung zu vermeiden, werden die Fehler zwar korrigiert und angestrichen, aber im Feedbacktext nicht erwähnt.

b) die Schülerinnen und Schüler einen standardisierten Rückmeldebogen erhalten, auf dem angekreuzt ist, was richtig und was falsch gemacht wurde. Das gibt den Schülerinnen und Schülern einen guten Überblick. Ferner ist das Feedback neutral, d.h. weder erfolgs- noch defizitorientiert.

c) die Schülerinnen und Schüler bei guten Leistungen oder wenn sie sich verbessert haben einen Stempel bekommen. Ein Stempel sagt in der Grundschule wesentlich mehr als Worte und motiviert die Schülerinnen und Schüler, sich anzustrengen, um einen solchen zu erwerben.

d) den Schülerinnen und Schülern verdeutlicht wird, was ihnen bereits gelungen ist; darüber hinaus sollten Fehler korrigiert werden und das Feedback einen Appell beinhalten, was konkret als nächstes getan werden soll, um die Leistung zu optimieren (individuelle Schwerpunktbildung).

e) die Fehler im Text lediglich angestrichen, aber keinesfalls korrigiert werden. So können die Schülerinnen und Schüler in der erneuten Auseinandersetzung mit dem Text aus ihren Fehlern lernen und diese eigenständig verbessern.

Quelle: Item weiterentwickelt aus Goldenstein (i.V.)

\section{Welche Aspekte prägen vorwiegend Bildungssprache?}

Sie können sich für eine oder mehrere Antworten entscheiden.

\begin{tabular}{|c|c|c|}
\hline a) Redundanzen & $\begin{array}{l}\text { g) unpersönliche Ausdrucks- } \\
\text { weise }\end{array}$ & O m) hoher Explizitheitsgrad \\
\hline b) komplexe Satzstrukturen & $\begin{array}{l}\text { O h) Verschmelzung von Funk- } \\
\text { tionswörtern }\end{array}$ & n) Nominalisierungen \\
\hline c) hohe Informationsdichte & i) komplexe Attribute & O o) Gesprächspartikel \\
\hline d) Ellipsen & j) reihende Satzstruktur & p) soziolektale Lexik \\
\hline e) Passivgebrauch & O k) Kontextgebundenheit & O q) mehrgliedrige Komposita \\
\hline f) geringe Lexikvarianz & O 1) Parenthesen & r) Fachbegriffe \\
\hline
\end{tabular}

Quellen: Koch \& Oesterreicher (1985, 1994); Morek \& Heller (2012); Goldenstein (i.V.) 


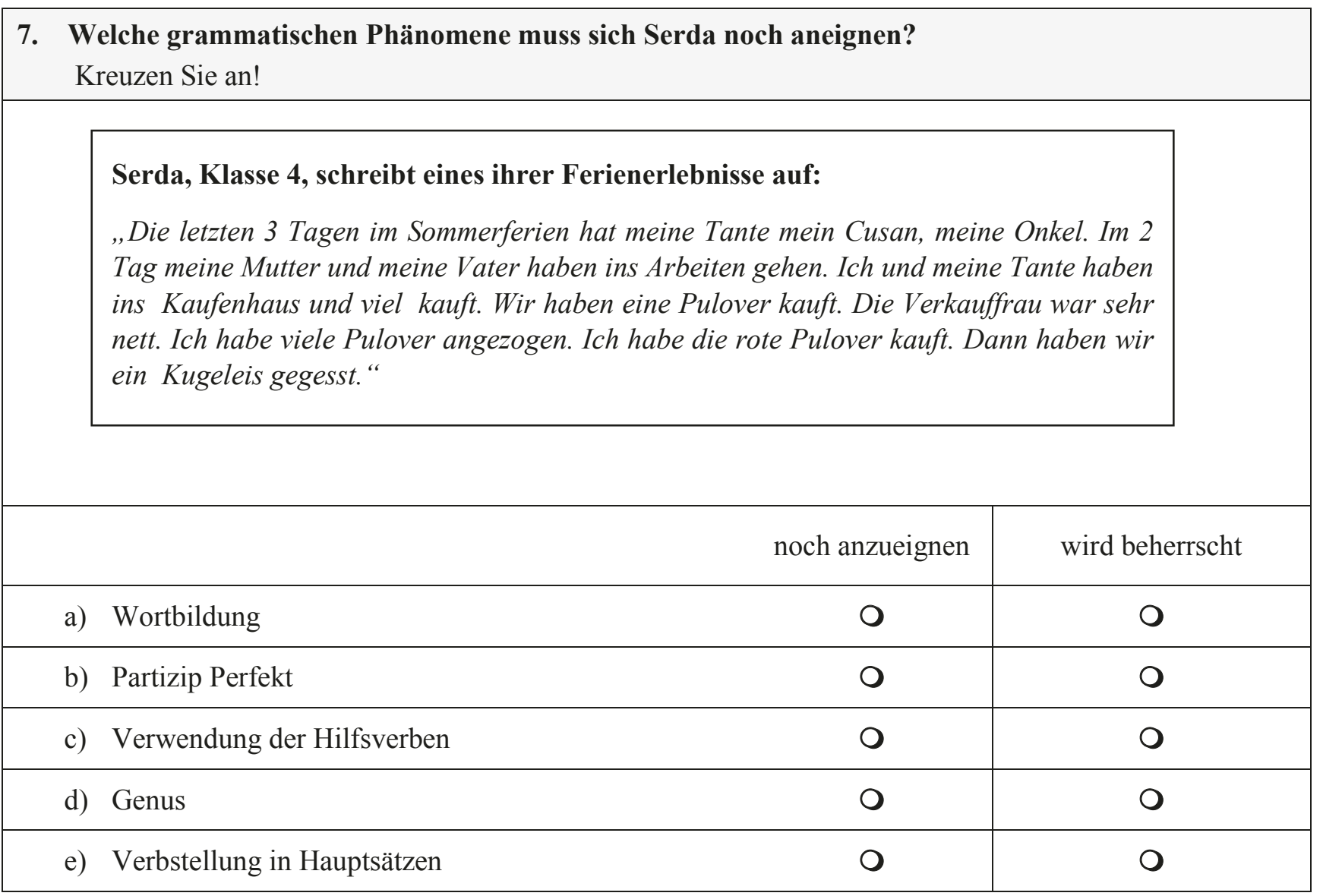

8. Welche Unterstützung ist für Anne die geeignetste, damit es ihr besser gelingt, einen grammatikalisch korrekten Text zu schreiben?

Sie können sich für genau eine Antwort entscheiden.

\section{Anne, Klasse 3, hat folgende Reizwortgeschichte geschrieben: \\ „Ich ging früh am morgen zum Garten und meine Eltern auch. Wir haben die Leiter geholt. Mein Papa flückte die Äpfel und ich helfe meiner Mama. Ich ging ganz langsam zu den wild Hasen, weil die haben Hunger und ich die fütern will. Mein Papa ist runtergefallt, weil der Ast ist gebrochen. Meine Mama und ich lachen. "}

a) Ich markiere alle Fehler und fordere sie auf, mit einem Mitschüler bzw. einer Mitschülerin, der/ die gut in Grammatik ist, den Text zu korrigieren. Danach prüfe ich den Text noch einmal.

b) In der Wochenplanarbeit bekommt Anne Übungsmaterial, um die Satzbildung selbstständig zu lernen. Zusätzlich erhält sie einen Lesetext, damit sie sich die Satzbildung implizit aneignet.

c) Ich spreche mit Anne über ausgewählte Fehler und übe mit ihr im Unterricht die Bildung von Haupt- und Nebensätzen.

d) Ich schreibe Annes Text einmal korrekt ab und bitte sie ihren Text mit meinem Text zu vergleichen. Danach soll sie meinen Text noch einmal in ihr Heft schreiben. 


\begin{tabular}{|c|c|c|c|}
\hline & \multicolumn{3}{|c|}{$\begin{array}{l}\text { „Ich war erst zu Hause... und hab mit meinem Bruder mit Bausteine gebaut, ... einen großen } \\
\text { Eiffelturm gebaut. Dann haben wir... ehm... mit zwei Hotwheel Autos so gemacht, ... damit... } \\
\text { der Auto drin geht, in den Eiffelturm. Dann haben wir son Garage macht... Dann, dann hab } \\
\text { ich so fest gemacht, ... dann ist alles kaputt gegangen. Dann sind wir rausgegangen. " }\end{array}$} \\
\hline & \multicolumn{3}{|l|}{ Quelle: Heilmann (2019) } \\
\hline \multirow[t]{3}{*}{ 9a) } & \multicolumn{3}{|c|}{$\begin{array}{l}\text { Welche sprachlichen Strukturen beherrscht der Schüler schon sicher? } \\
\text { Sie können sich für eine oder mehrere Antworten entscheiden. }\end{array}$} \\
\hline & O a) Gebrauch der Inversion & o c) Genus & $\mathrm{O}$ e) Verbstellung in Nebensätzen \\
\hline & O b) Kasus nach Präpositionen & d) Wortstellung & f) Satzklammer \\
\hline \multirow[t]{3}{*}{ 9b) } & \multicolumn{3}{|c|}{$\begin{array}{l}\text { Welches nächste vorrangige Ziel setzen Sie dem Kind in seinem Sprachaneignungsprozess? } \\
\text { Sie können sich für genau eine Antwort entscheiden. }\end{array}$} \\
\hline & $\begin{array}{l}\text { a) Ausdifferenzierung des } \\
\text { Wortschatzes }\end{array}$ & $\begin{array}{l}\text { c) Verkettung von Äußer- } \\
\text { rungen }\end{array}$ & e) korrekte Flexion \\
\hline & $\begin{array}{l}\text { b) variantenreichere Satz- } \\
\text { strukturen }\end{array}$ & $\begin{array}{l}\text { d) Sicherheit im Tempus- } \\
\text { gebrauch }\end{array}$ & $\begin{array}{l}\text { f) Verwendung von Attri- } \\
\text { buten }\end{array}$ \\
\hline
\end{tabular}

10. Im Folgenden sind verschiedene Thesen zum sprachlichen Lernen eines Kindes aufgeführt. Kreuzen Sie an, in welchem Ausmaß Sie diesen zustimmen.

a) Die sprachliche Kompetenz eines Kindes wird durch das Vorlesen gefördert.

b) Zuerst benötigt das Kind einen umfangreichen Wortschatz, danach kann es grammatische Strukturen erlernen.

c) Wenn in der Familie vor allem Dialekt gesprochen wird, erschwert dies die Aneignung der Schriftsprache.

d) Eine hohe Sprachkompetenz hat vor allem etwas mit Begabung zu tun.

e) Kinder mit geringem Wortschatz müssen zuallererst die Wörter lernen. Erst danach kommt alles andere.

f) Das Hören von Hörspielen und Hörgeschichten wirkt sich positiv auf die Sprachentwicklung aus.

g) Kinder mit anderen Erstsprachen als Deutsch brauchen generell länger, um sich Sprachkompetenzen im Deutschen anzueignen. 


\section{Welche Aussagen über „Scaffolding“ treffen zu?}

Sie können sich für eine oder mehrere Antworten entscheiden.

a) Scaffolding wird den konstruktivistischen Lerntheorien zugeordnet.

b) Scaffolding ist eine Technik, welche spezifisch für die Sprachförderung entwickelt wurde, um alltagssprachliche Formulierungen der Schülerinnen und Schüler zu erweitern.

c) Beim Scaffolding ist es gewünscht, dass der Lernende sich möglichst selbstständig dem Ziel nähert. Dabei werden Um- oder Irrwege in Kauf genommen. Es wird davon ausgegangen, dass diese Um-/Irrwege Negativwissen erzeugen, welches ebenfalls als Lernfortschritt erachtet wird.

d) Scaffolding bezeichnet im pädagogisch-psychologischen Kontext die Unterstützung des Lernprozesses durch die Bereitstellung einer ersten vollständigen Orientierungsgrundlage in Form von Anleitungen, Denkanstößen und anderen Hilfestellungen.

e) Beim Scaffolding wird mit steigender Erfahrung und besserem Verständnis der Schülerinnen und Schüler die Komplexität der Aufgaben immer weiter erhöht und die Unterstützung schrittweise verringert.

f) Scaffolding wird in der Literatur häufig auch als „entdeckendes Lernen“ bezeichnet.

g) Beim Scaffolding steuert die Lehrkraft aktiv, welcher Schüler bzw. welche Schülerin welche Hilfestellungen benötigt bzw. sie leitet den Abbau der Hilfestellungen in Orientierung an die Zone der proximalen Entwicklung an.

h) Bei der Durchführung von Scaffolding spielen die Aspekte Modelling, Assisting und Monitoring eine wichtige Rolle.

Quelle: Quehl (2010) 
12. Im Folgenden lesen Sie die mündliche Erklärung einer Drittklässlerin zum Thema „Der Weg der Nahrung durch den Körper". Welche Formulierungen enthalten bildungssprachliche Elemente?

Unterstreichen Sie die Elemente im Text und benennen Sie das jeweilige Element rechts mit seinem Fachbegriff.

Erst beißen wir ein Stück vom Brot ab. Durch das Kauen machen wir es klein.

Danach rutscht die Nahrung die Speiseröhre herunter in den Magen und wird mit den Magensäften vermischt.

Dann gelangt die Nahrung in den Pförtner und in den Dünndarm.

Dort bringt das Blut die Bausteine in den richtigen Ort.

Im Dickdarm wird der ganze Saft rausgezogen.

Danach landet die Nahrung in der Kloschüssel.

bildungssprachliche Elemente

Quelle: Item weiterentwickelt aus Fornol (2019) 


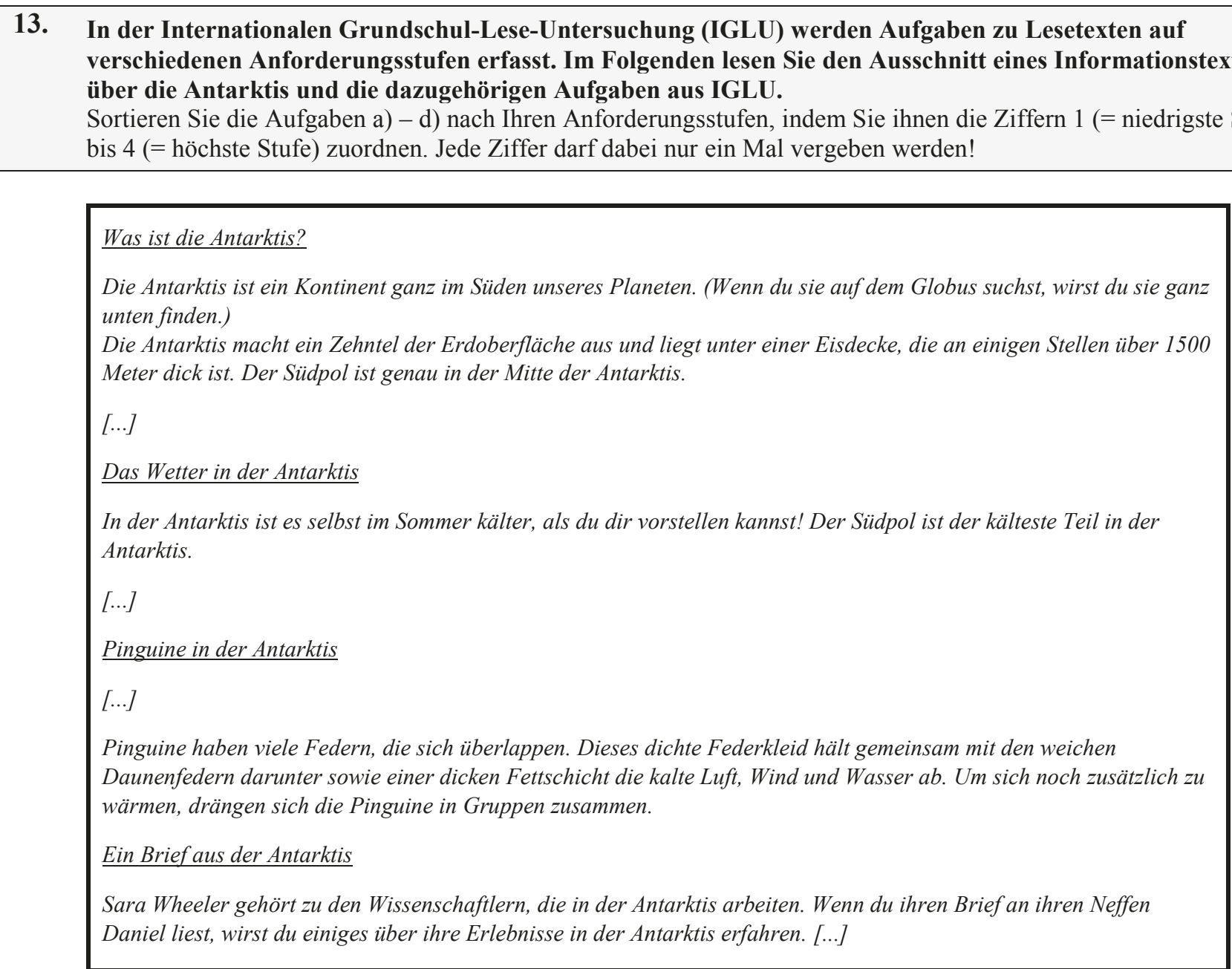

\section{Aufgaben}

a) Nenne drei Wege, wie sich die Pinguine in der Antarktis warm halten. 1. 2. 3.

b) Würdest du gerne in die Antarktis reisen? Benutze das, was du in beiden Texten In der Antarktis und Ein Brief aus der Antarktis gelesen hast, um deine Meinung zu begründen.

c) Wo kannst du die Antarktis auf dem Globus finden?

d) In welchem Teil des Textes steht, wie dick das Eis in der Antarktis ist?
A. Was ist die Antarktis?
B. Das Wetter in der Antarktis
C. Pinguine in der Antarktis
D. Ein Brief aus der Antarktis

\section{Anforderungsstufe}

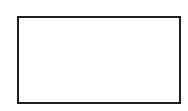

Quelle: Item weiterentwickelt aus Bos et al. (2007) und Goldenstein (i.V.) 
14. Was können Sie tun, um Simons Sprechanteile zu erhöhen?

Sie können sich für eine oder mehrere Antworten entscheiden.

\section{Alex und Simon (Klasse 2) haben sich in Partnerarbeit Wissen über das Meerschweinchen angeeignet. Nun präsentieren sie zusammen ihre Ergebnisse:}

Alex: „Also, wir haben etwas über „,Meerschweinchen“ herausgefunden.

Meerschweinchen sind nämlich gar keine Schweine, sondern Nager. "

Simon: ,, Genau, das sind keine Schweine. “

Alex: „Meerschweinchen kommen aus Mittel- und Südamerika. Bei den Inkas waren sie auch schon Haustiere. Aber, sie wurden auch gegessen. “

Simon: , Genau, die haben sie gegessen. “

Alex: „In Deutschland werden Meerschweinchen nicht gegessen. Hier sind sie nur Haustiere. Wichtig ist, dass Meerschweinchen nicht allein sein wollen. Die brauchen Artgenossen, also andere Meerschweinchen."

Simon: ,Genau, andere Meerschweinchen. “

a) Alex und Simon sollen ihre Ergebnisse zuerst mir vortragen. Ich korrigiere sie dann während des Vortragens. So kommt später auch Simon zu Wort, wenn sie vor der Klasse sprechen.

b) Alex und Simon bekommen die gleiche Anzahl an leeren Vortragskarten. Sie sollen darauf notieren, was sie vortragen wollen. Danach üben sie das Vortragen zuerst für sich.

c) Ich biete Vortragskarten mit sprachlichen Mustern an, die die Schüler bei Bedarf nutzen können.

d) Ich unterbreche den Vortrag an geeigneter Stelle und gebe Simon den Hinweis, sich mehr einzubringen. 
15. Welche Lernvoraussetzungen müssen Drittklässler mitbringen, um einen nachvollziehbaren Kurzvortrag zu gestalten? Kreuzen Sie an, in welchem Ausmaß Sie zustimmen.

a) Kinder benötigen sprachliche Redemittel und Wendungen, um sich mitteilen zu können.

b) Kinder müssen grammatikalisch korrekt sprechen können.

c) Kinder müssen sich verständlich ausdrücken können.

O

O

d) Kinder müssen über einen sehr guten Wortschatz verfügen.

e) Kinder müssen grundlegende Fachbegriffe kennen.

f) Kinder sollten sich trauen, vor anderen zu sprechen.

16. Welche Unterstützungsmaßnahmen können Schülerinnen und Schülern das mündliche Vortragen erleichtern? Sie können sich für eine oder mehrere Antworten entscheiden.

a) Simulation/Vorbereitung in Kleingruppen O

b) zur Verfügung stellen von sprachlichen Wendungen O

c) Vortrag auswendig lernen lassen

d) Erstellen ausformulierter Moderationskarten

e) freie Themenwahl

f) Einüben kurzer Statements 


\section{Worauf führen Sie die grammatikalischen Fehler des Zweitklässlers zurück?}

Sie können sich für genau eine Antwort entscheiden.

\section{Noah berichtet Oliver in einem Brief aus dem Urlaub begeistert von seinem neuen Traumberuf:}

\section{„Lieber Oliver,}

Ich will ein Meresbiologe sein! Ich war im Fischmuseum und da arbeiten viele Meresbiologen. Ein Meresbiologe erfoscht das Meer, die Pflantzen und die Vische die da leben. Er muss tauchen viele herum, damit er sie findet. Es ist ser gefärlich! Ein Hei ist ein ser große Visch. Über dem Wasser du siehst echt nur die Vlosse von dem Hei! Der Hei ist sehr schnell und kann fressen soga ein Mensch! Ich liebe Abenteur und wilde Tiere! Das ist warum ich ein super Meresbiologe bin!

Ich möchte jetzt wissen was du machest später

Dein Noah!"

a) Noah leidet vermutlich an Legasthenie und hat daher sonderpädagogischen Förderbedarf.

b) Es handelt sich um typische Probleme beim Schriftspracherwerb im Anfangsunterricht.

c) Die Fehler lassen sich auf eine dialektal gefärbte Familiensprache zurückführen.

d) Der Schüler befindet sich im Zweitspracherwerb.

e) Der Schüler könnte infolge einer Beeinträchtigung des Gehörs eine Sprachstörung aufweisen. Ein Facharzt sollte die Vermutung abklären. 
2. Kodiermanual für quantitative Aufgaben 


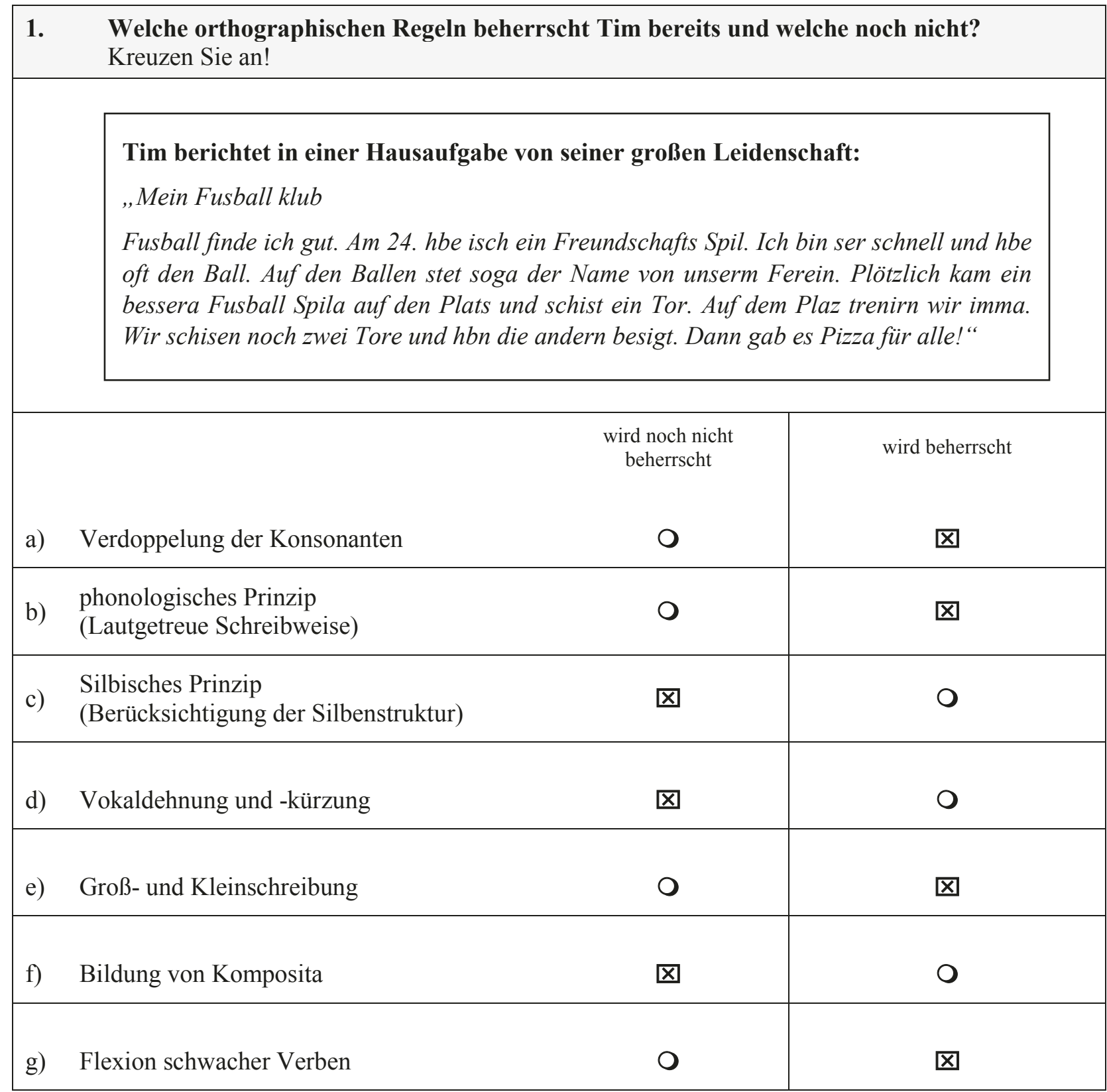

\section{Kodierung:}

- Der Score bei Item 1 ergibt sich als Division der Summe der korrekt angekreuzten Antwortkategorien geteilt durch den Wert 3,5. Somit resultiert ein maximaler Score von 2 Punkten.

- Bei falsch angekreuzten Antwortkategorien erfolgt kein Punktabzug.

- Wurde keine der beiden Antwortoptionen ausgewählt: 0 Punkte für die betroffene Antwortkategorie. 


\section{Bitte lesen Sie den folgenden Sachtext, der für eine dritte Klasse bestimmt ist!}

\section{Knochen und Gelenke}

Die Knochen schützen und stützen den Körper. Ein Knochen besteht aus vielen tausend Knochenzellen, die wie alle Zellen von Blutgefäßen mit Sauerstoff und Nährsalzen versorgt werden. Umgekehrt geben sie aber auch Abfallstoffe ins Blut ab.

Die dünne äußere Hülle nennt man Knochenhaut. Sie erfüllt eine wichtige Funktion beim Wachstum und der Erneuerung (Regeneration) der Knochen. Im Inneren der großen Knochen befindet sich das Knochenmark. Dort werden die roten und weißen Blutzellen gebildet. Am Skelett setzen außerdem auch die Muskeln an.

Gelenke und Muskeln ermöglichen die Bewegung des Körpers. In einem Gelenk treffen zwei oder mehrere Knochen zusammen. Es gibt verschiedene Arten von Gelenken. Das Scharniergelenk sorgt zum Beispiel dafür, dass wir unser Knie beim Hinsetzen beugen können.

Jeder Mensch, also auch du, besitzt über 100 Gelenke und etwa 206 Knochen. Der kleinste Knochen ist der Steigbügel im Ohr und der größte ist der Oberschenkelknochen.

2a) Welche Merkmale des Textes können das Leseverstehen erschweren? Identifizieren Sie mögliche sprachliche Barrieren!

$\bullet$
$\bullet$
-

2b) Welche Hilfen würden Sie für Schülerinnen und Schüler anbieten, die schon häufig Probleme beim Leseverstehen zeigten?

Sie können sich für eine oder mehrere Antworten entscheiden.

a) Ich stelle Informationstexte zur Verfügung, welche die verwendeten Fachbegriffe erläutern. Dazu können z.B. Lexika oder Sachbücher genutzt werden.

b) Ich ergänze den Text um anatomische Abbildungen, die den Inhalt graphisch darstellen. Die 区 Kombination von Text und Bild kann auf diese Weise das Verstehen unterstützen.

c) Ich streiche die meisten Fachbegriffe und kürze den Text in seiner Länge, um den Schwierigkeitsgrad zu reduzieren.

Ich markiere Schlüsselwörter, vergrößere die Schrift sowie den Zeilenabstand und unterstreiche die wichtigsten Informationen, um die Identifikation von zentralen Wörtern und Sätzen zu erleichtern.

\section{Kodierung:}

- Der Score bei Item $2 b$ ergibt sich als Division der Summe der korrekt angekreuzten Antwortkategorien geteilt durch den Wert 2. Somit resultiert ein maximaler Score von 2 Punkten.

- Bei falsch angekreuzten Antwortkategorien erfolgt kein Punktabzug.

- Wurde keine der Antwortkategorien ausgewählt, beträgt der Score des Items 0 Punkte. 


\begin{tabular}{|c|}
\hline 3. Nennen Sie Lesestrategien für den weiterführenden Leseunterricht (nicht: Leselernmethoden)! \\
\hline$\bullet$ \\
$\qquad$ \\
S. Kodiermanual für qualitative \\
Aufgaben! \\
\hline
\end{tabular}

4. Dhakiya ist vor einem Jahr nach Deutschland gekommen. Nun besucht sie die dritte Klasse und schreibt über ihren neunten Geburtstag, den sie vor zwei Wochen feierte.

Wie können Sie die Schülerin ausgehend von diesem Text fördern?

Sie können sich für eine oder mehrere Antworten entscheiden.

„Mein Geburtstag
Morgens gab es viele Pakete. In den Paketen waren viel schöne Sachen. In der Schule die
anderen Kinder sangen ein Lid für mich. Das freute mich!
Nachmittags andere Kinder kamen zu mir nach Hause. Meine Mutter backte einen
schönen Kuchen. Die Kinder und ich spielten viel. Das war Luchsus!
Abends ging ich schlafen und der Tag war vorbei. Das war schade! Ich möchte haben
jeden Tag Geburtstag!“"

a) Ich kennzeichne Rechtschreib- und Grammatikfehler sowie Wortwiederholungen, damit die Schülerin diese selbstständig mit einem Wörterbuch verbessern kann.

b) Ich schreibe der Schülerin unter ihren Text, was sie bereits gut kann und bekräftige sie auf diese Weise in ihren Bemühungen. Die Fehler lasse ich unberücksichtigt, um Frustrationserlebnisse zu vermeiden.

c) Ich biete der Schülerin spezielle Grammatikübungen zur Verbstellung im Deutschen an, damit sie ihre grammatischen Kenntnisse vertiefen kann. Im Anschluss daran bitte ich sie, ihren Text erneut durchzusehen.

d) Ich bitte im Sinne des „Peer-Tutoring“ einen besonders leistungsstarken Mitschüler bzw. eine Mitschülerin den Text zu korrigieren und ziehe mich zurück. Kinder finden meist einen besseren Zugang zueinander, was sich positiv auf den Lernprozess auswirkt.

\section{Kodierung:}

- Der Score bei Item 4 ergibt sich als Division der Summe der korrekt angekreuzten Antwortkategorien geteilt durch den Wert 2. Somit resultiert ein maximaler Score von 2 Punkten.

- Bei falsch angekreuzten Antwortkategorien erfolgt kein Punktabzug.

- Wurde keine der Antwortkategorien ausgewählt, beträgt der Score des Items 0 Punkte. 


\section{Feedback ist bei schriftlichen Texten für ein Kind dann besonders förderlich, wenn...}

Sie können sich für genau eine Antwort entscheiden.

a) sich insbesondere darauf konzentriert wird, was dem Kind schon gut gelungen ist. Um eine Defizitorientierung zu vermeiden, werden die Fehler zwar korrigiert und angestrichen, aber im Feedbacktext nicht erwähnt.

b) die Schülerinnen und Schüler einen standardisierten Rückmeldebogen erhalten, auf dem angekreuzt ist, was richtig und was falsch gemacht wurde. Das gibt den Schülerinnen und Schülern einen guten Überblick. Ferner ist das Feedback neutral, d.h. weder erfolgs- noch defizitorientiert.

c) die Schülerinnen und Schüler bei guten Leistungen oder wenn sie sich verbessert haben einen Stempel bekommen. Ein Stempel sagt in der Grundschule wesentlich mehr als Worte und motiviert die Schülerinnen und Schüler, sich anzustrengen, um einen solchen zu erwerben.

d) den Schülerinnen und Schülern verdeutlicht wird, was ihnen bereits gelungen ist; darüber hinaus sollten Fehler korrigiert werden und das Feedback einen Appell beinhalten, was konkret als nächstes getan werden soll, um die Leistung zu optimieren (individuelle Schwerpunktbildung).

e) die Fehler im Text lediglich angestrichen, aber keinesfalls korrigiert werden. So können O die Schülerinnen und Schüler in der erneuten Auseinandersetzung mit dem Text aus ihren Fehlern lernen und diese eigenständig verbessern.

Quelle: Item weiterentwickelt aus Goldenstein (i.V.)

\section{Kodierung:}

- Wurde die richtige Antwortkategorie in Item 5 angekreuzt, beträgt der Score 1 Punkt. Somit resultiert ein maximaler Score von 1 Punkt.

- Bei falsch angekreuzten Antwortkategorien erfolgt kein Punktabzug.

- Wurden mehrere oder keine Antwortkategorie(n) angekreuzt, beträgt der Score des Items 0 Punkte. 


\section{Welche Aspekte prägen vorwiegend Bildungssprache?}

Sie können sich für eine oder mehrere Antworten entscheiden.

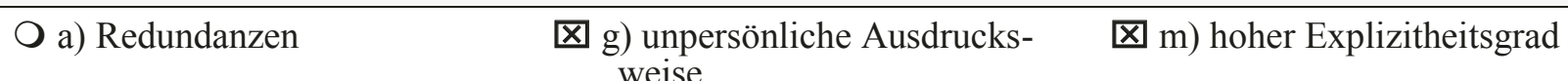

凶) komplexe Satzstrukturen

h) Verschmelzung von Funktionswörtern

凶n) Nominalisierungen

凶 c) hohe Informationsdichte

凶 i) komplexe Attribute

o) Gesprächspartikel

d) Ellipsen

j) reihende Satzstruktur

p) soziolektale Lexik

凶e) Passivgebrauch

k) Kontextgebundenheit

凶) mehrgliedrige Komposita

f) geringe Lexikvarianz

1) Parenthesen

凶 r) Fachbegriffe

Quellen: Koch \& Oesterreicher (1985, 1994); Morek \& Heller (2012); Goldenstein (i.V.)

\section{Kodierung:}

- Der Score bei Item 6 ergibt sich als Division der Summe der korrekt angekreuzten Antwortkategorien geteilt durch den Wert 9. Somit resultiert ein maximaler Score von 2 Punkten.

- Bei falsch angekreuzten Antwortkategorien erfolgt kein Punktabzug.

- Wurde keine der Antwortkategorien ausgewählt, beträgt der Score des Items 0 Punkte. 


\begin{tabular}{|c|c|c|c|}
\hline \multicolumn{4}{|c|}{$\begin{array}{l}\text { 7. Welche grammatischen Phänomene muss sich Serda noch aneignen? } \\
\text { Kreuzen Sie an! }\end{array}$} \\
\hline & \multicolumn{3}{|c|}{$\begin{array}{l}\text { Serda, Klasse 4, schreibt eines ihrer Ferienerlebnisse auf: } \\
\text { „Die letzten } 3 \text { Tagen im Sommerferien hat meine Tante mein Cusan, meine Onkel. Im } 2 \\
\text { Tag meine Mutter und meine Vater sind ins Arbeiten gehen. Ich und meine Tante haben } \\
\text { ins Kaufenhaus und viel kauft. Wir haben eine Pulover kauft. Die Verkauffrau war sehr } \\
\text { nett. Ich habe viele Pulover angezogen. Ich habe die rote Pulover kauft. Dann haben wir } \\
\text { ein Kugeleis gegesst.“ }\end{array}$} \\
\hline & & noch anzueignen & wird beherrscht \\
\hline a) & Wortbildung & 凶 & $\mathrm{O}$ \\
\hline b) & Partizip Perfekt & 凶 & O \\
\hline c) & Verwendung der Hilfsverben & O & 凶 \\
\hline & Genus & 凶 & 0 \\
\hline e) & Verbstellung in Hauptsätzen & 凶 & 0 \\
\hline
\end{tabular}

\section{Kodierung:}

- Der Score bei Item 7 ergibt sich als Division der Summe der korrekt angekreuzten Antwortkategorien geteilt durch den Wert 2,5. Somit resultiert ein maximaler Score von 2 Punkten.

- Bei falsch angekreuzten Antwortkategorien erfolgt kein Punktabzug.

- Wurde keine der beiden Antwortoptionen ausgewählt: 0 Punkte für die betroffene Antwortkategorie. 
8. Welche Unterstützung ist für Anne die geeignetste, damit es ihr besser gelingt, einen grammatikalisch korrekten Text zu schreiben?

Sie können sich für genau eine Antwort entscheiden.

Anne, Klasse 3, hat folgende Reizwortgeschichte geschrieben:

„Ich ging früh am morgen zum Garten und meine Eltern auch. Wir haben die Leiter geholt.

Mein Papa flückte die Äpfel und ich helfe meiner Mama. Ich ging ganz langsam zu den wild

Hasen, weil die haben Hunger und ich die fütern will. Mein Papa ist runtergefallt, weil der Ast ist gebrochen. Meine Mama und ich lachen. "

a) Ich markiere alle Fehler und fordere sie auf, mit einem Mitschüler bzw. einer Mitschülerin, der/ die gut in Grammatik ist, den Text zu korrigieren. Danach prüfe ich den Text noch einmal.

b) In der Wochenplanarbeit bekommt Anne Übungsmaterial, um die Satzbildung selbstständig zu lernen. Zusätzlich erhält sie einen Lesetext, damit sie sich die Satzbildung implizit aneignet.

c) Ich spreche mit Anne über ausgewählte Fehler und übe mit ihr im Unterricht die Bildung von Haupt- und Nebensätzen.

d) Ich schreibe Annes Text einmal korrekt ab und bitte sie ihren Text mit meinem Text zu vergleichen. Danach soll sie meinen Text noch einmal in ihr Heft schreiben.

\section{Kodierung:}

- Wurde die richtige Antwortkategorie in Item 8 angekreuzt, beträgt der Score 1 Punkt. Somit resultiert ein maximaler Score von 1 Punkt.

- Bei falsch angekreuzten Antwortkategorien erfolgt kein Punktabzug.

- Wurden mehrere oder keine Antwortkategorie(n) angekreuzt, beträgt der Score des Items 0 Punkte.

\section{Lesen Sie die nachstehende mündliche Schüleräußerung.}

„Ich war erst zu Hause... und hab mit meinem Bruder mit Bausteine gebaut, ... einen großen
Eiffelturm gebaut. Dann haben wir... ehm ... mit zwei Hotwheel Autos so gemacht, ... damit...
der Auto drin geht, in den Eiffelturm. Dann haben wir son Garage macht... Dann, dann hab
ich so fest gemacht, ... dann ist alles kaputt gegangen. Dann sind wir rausgegangen. “

Quelle: Heilmann (2019)

9a) Welche sprachlichen Strukturen beherrscht der Schüler schon sicher? Sie können sich für eine oder mehrere Antworten entscheiden.
冈) Gebrauch der Inversion
c) Genus
凶 e) Verbstellung in Nebensätzen
O b) Kasus nach Präpositionen
囚 d) Wortstellung
凶) Satzklammer

\section{Kodierung:}

- Der Score bei Item 9a ergibt sich als Division der Summe der korrekt angekreuzten Antwortkategorien geteilt durch den Wert $\underline{3}$. Somit resultiert ein maximaler Score von 2 Punkten.

- Bei falsch angekreuzten Antwortkategorien erfolgt kein Punktabzug.

- Wurde keine der Antwortkategorien ausgewählt, beträgt der Score des Items 0 Punkte. 
9b) Welches nächste vorrangige Ziel setzen Sie dem Kind in seinem Sprachaneignungsprozess? Sie können sich für genau eine Antwort entscheiden.
囚 a) Ausdifferenzierung des Wortschatzes
O c) Verkettung von Äußer- rungen
b) variantenreichere Satz- strukturen
O) Sicherheit im Tempus- gebrauch
e) korrekte Flexion
f) Verwendung von Attri- buten

\section{Kodierung:}

- Wurde die richtige Antwortkategorie in Item $9 b$ angekreuzt, beträgt der Score 1 Punkt. Somit resultiert ein maximaler Score von 1 Punkt.

- Bei falsch angekreuzten Antwortkategorien erfolgt kein Punktabzug.

- Wurden mehrere oder keine Antwortkategorie(n) angekreuzt, beträgt der Score des Items 0 Punkte

10. Im Folgenden sind verschiedene Thesen zum sprachlichen Lernen eines Kindes aufgeführt. Kreuzen Sie an, in welchem Ausmaß Sie diesen zustimmen.

a) Die sprachliche Kompetenz eines Kindes wird durch das
Vorlesen gefördert.
b) Zuerst benötigt das Kind einen umfangreichen Wortschatz,
danach kann es grammatische Strukturen erlernen.

\section{Kodierung:}

- Der Score bei Item 10 ergibt sich als Division der Summe der korrekt angekreuzten Antwortkategorien geteilt durch den Wert 3,5. Somit resultiert ein maximaler Score von 2 Punkten.

- Bei falsch angekreuzten Antwortkategorien erfolgt kein Punktabzug.

- Wurde keine der beiden Antwortoptionen ausgewählt: 0 Punkte für die betroffene Antwortkategorie. 


\section{Welche Aussagen über „Scaffolding“ treffen zu?}

Sie können sich für eine oder mehrere Antworten entscheiden.

a) Scaffolding wird den konstruktivistischen Lerntheorien zugeordnet.

b) Scaffolding ist eine Technik, welche spezifisch für die Sprachförderung entwickelt wurde, um alltagssprachliche Formulierungen der Schülerinnen und Schüler zu erweitern.

c) Beim Scaffolding ist es gewünscht, dass der Lernende sich möglichst selbstständig dem Ziel nähert. Dabei werden Um- oder Irrwege in Kauf genommen. Es wird davon ausgegangen, dass diese Um-/Irrwege Negativwissen erzeugen, welches ebenfalls als Lernfortschritt erachtet wird.

d) Scaffolding bezeichnet im pädagogisch-psychologischen Kontext die Unterstützung des Lernprozesses durch die Bereitstellung einer ersten vollständigen Orientierungsgrundlage in Form von Anleitungen, Denkanstößen und anderen Hilfestellungen.

e) Beim Scaffolding wird mit steigender Erfahrung und besserem Verständnis der Schülerinnen und Schüler die Komplexität der Aufgaben immer weiter erhöht und die Unterstützung schrittweise verringert.

f) Scaffolding wird in der Literatur häufig auch als „entdeckendes Lernen“ bezeichnet.

g) Beim Scaffolding steuert die Lehrkraft aktiv, welcher Schüler bzw. welche Schülerin 凶 welche Hilfestellungen benötigt bzw. sie leitet den Abbau der Hilfestellungen in Orientierung an die Zone der proximalen Entwicklung an.

h) Bei der Durchführung von Scaffolding spielen die Aspekte Modelling, Assisting und Monitoring eine wichtige Rolle.

Quelle: Quehl (2010)

\section{Kodierung:}

- Der Score bei Item 11 ergibt sich als Division der Summe der korrekt angekreuzten Antwortkategorien geteilt durch den Wert 4. Somit resultiert ein maximaler Score von 2 Punkten.

- Bei falsch angekreuzten Antwortkategorien erfolgt kein Punktabzug.

- Wurde keine der Antwortkategorien ausgewählt, beträgt der Score des Items 0 Punkte. 
12. Im Folgenden lesen Sie die mündliche Erklärung einer Drittklässlerin zum Thema „Der Weg der Nahrung durch den Körper". Welche Formulierungen enthalten bildungssprachliche Elemente? Unterstreichen Sie die Elemente im Text und benennen Sie das jeweilige Element rechts mit seinem Fachbegriff.

Es genügt das Erkennen der vier Elemente „Nominalisierung“, „Fachbegriff / Fachwortschatz“", „Kompositum“ und „Passivkonstruktionen“ mit je einem konkreten Beispiel!

Erst beißen wir ein Stück vom Brot ab. Durch das Kauen machen wir es klein.

Danach rutscht die Nahrung die Speiseröhre herunter in den Magen und wird mit den Magensäften vermischt.

Dann gelangt die Nahrung in den Pförtner und in den Dünndarm. Fachbegriffe + Kompositum)

Dort bringt das Blut die Bausteine in den richtigen Ort.

Im Dickdarm wird der ganze Saft rausgezogen.

(weiteres Bsp. für Fachbegriffe + Passiv

+ Kompositum)

Danach landet die Nahrung in der Kloschüssel.

Quelle: Item weiterentwickelt aus Fornol (2019)

Kodierung:

- Pro korrekte Unterstreichung und korrekte Benennung wird 1 Punkt vergeben (keine Vergabe von halben Punkten).

- Der Score bei Item 12 ergibt sich als Division der Summe der korrekten Nennungen (und Unterstreichungen) geteilt durch den Wert 2. Somit resultiert ein maximaler Score von 2 Punkten.

- Bei falscher Nennung erfolgt kein Punktabzug.

- Wurde die Aufgabe nicht bearbeitet, beträgt der Score 0 Punkte. 
13. In der Internationalen Grundschul-Lese-Untersuchung (IGLU) werden Aufgaben zu Lesetexten auf verschiedenen Anforderungsstufen erfasst. Im Folgenden lesen Sie den Ausschnitt eines Informationstextes über die Antarktis und die dazugehörigen Aufgaben aus IGLU.

Sortieren Sie die Aufgaben a) - d) nach Ihren Anforderungsstufen, indem Sie ihnen die Ziffern 1 (= niedrigste Stufe) bis 4 (= höchste Stufe) zuordnen. Jede Ziffer darf dabei nur ein Mal vergeben werden!

Was ist die Antarktis?

Die Antarktis ist ein Kontinent ganz im Süden unseres Planeten. (Wenn du sie auf dem Globus suchst, wirst du sie ganz unten finden.)

Die Antarktis macht ein Zehntel der Erdoberfläche aus und liegt unter einer Eisdecke, die an einigen Stellen über 1500 Meter dick ist.

Der Südpol ist genau in der Mitte der Antarktis.

$[\ldots]$

Das Wetter in der Antarktis

In der Antarktis ist es selbst im Sommer kälter, als du dir vorstellen kannst! Der Südpol ist der kälteste Teil in der Antarktis.

$[\ldots]$

Pinguine in der Antarktis

[...]

Pinguine haben viele Federn, die sich überlappen. Dieses dichte Federkleid hält gemeinsam mit den weichen Daunenfedern darunter sowie einer dicken Fettschicht die kalte Luft, Wind und Wasser ab. Um sich noch zusätzlich zu wärmen, drängen sich die Pinguine in Gruppen zusammen.

Ein Brief aus der Antarktis

Sara Wheeler gehört zu den Wissenschaftlern, die in der Antarktis arbeiten. Wenn du ihren Brief an ihren Neffen Daniel liest, wirst du einiges über ihre Erlebnisse in der Antarktis erfahren.

$[\cdots]$

\section{Aufgaben}

a) Nenne drei Wege, wie sich die Pinguine in der Antarktis warm halten.

1 .

2.

3.

b) Würdest du gerne in die Antarktis reisen? Benutze das, was du in beiden Texten In der Antarktis und Ein Brief aus der Antarktis gelesen hast, um deine Meinung zu begründen.

c) Wo kannst du die Antarktis auf dem Globus finden?

d) In welchem Teil des Textes steht, wie dick das Eis in der Antarktis ist?

A. Was ist die Antarktis?

B. Das Wetter in der Antarktis

C. Pinguine in der Antarktis

D. Ein Brief aus der Antarktis
Anforderungsstufe

2

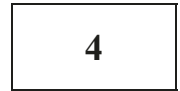

1

3

Quelle: Item weiterentwickelt aus Bos et al. (2007) und Goldenstein (i.V.)

\section{Kodierung:}

- Der Score bei Item 13 ergibt sich als Division der Summe der korrekten Ziffern geteilt durch den Wert 2. Somit resultiert ein maximaler Score von 2 Punkten.

- Bei fehlenden oder falschen Ziffern erfolgt kein Punktabzug. 
14. Was können Sie tun, um Simons Sprechanteile zu erhöhen?

Sie können sich für eine oder mehrere Antworten entscheiden.

\section{Alex und Simon (Klasse 2) haben sich in Partnerarbeit Wissen über das \\ Meerschweinchen angeeignet. Nun präsentieren sie zusammen ihre Ergebnisse:}

Alex: „Also, wir haben etwas über „,Meerschweinchen“ herausgefunden.

Meerschweinchen sind nämlich gar keine Schweine, sondern Nager. “

Simon: ,, Genau, das sind keine Schweine. “

Alex: „Meerschweinchen kommen aus Mittel- und Südamerika. Bei den Inkas waren sie auch schon Haustiere. Aber, sie wurden auch gegessen. “

Simon: „, Genau, die haben sie gegessen. “

Alex: „In Deutschland werden Meerschweinchen nicht gegessen. Hier sind sie nur Haustiere. Wichtig ist, dass Meerschweinchen nicht allein sein wollen. Die brauchen Artgenossen, also andere Meerschweinchen. "

Simon: „, Genau, andere Meerschweinchen. “

a) Alex und Simon sollen ihre Ergebnisse zuerst mir vortragen. Ich korrigiere sie dann während des Vortragens. So kommt später auch Simon zu Wort, wenn sie vor der Klasse sprechen.

b) Alex und Simon bekommen die gleiche Anzahl an leeren Vortragskarten. Sie sollen darauf notieren, was sie vortragen wollen. Danach üben sie das Vortragen zuerst für sich.

c) Ich biete Vortragskarten mit sprachlichen Mustern an, die die Schüler bei Bedarf nutzen können.

d) Ich unterbreche den Vortrag an geeigneter Stelle und gebe Simon den Hinweis, sich mehr einzubringen.

\section{Kodierung:}

- Der Score bei Item 14 ergibt sich als Division der Summe der korrekt angekreuzten Antwortkategorien geteilt durch den Wert 2. Somit resultiert ein maximaler Score von 2 Punkten.

- Bei falsch angekreuzten Antwortkategorien erfolgt kein Punktabzug.

- Wurde keine der Antwortkategorien ausgewählt, beträgt der Score des Items 0 Punkte. 
15. Welche Lernvoraussetzungen müssen Drittklässler mitbringen, um einen nachvollziehbaren Kurzvortrag zu gestalten? Kreuzen Sie an, in welchem Ausmaß Sie zustimmen.

g) Kinder benötigen sprachliche Redemittel und

Wendungen, um sich mitteilen zu können.

h) Kinder müssen grammatikalisch korrekt sprechen

凶

O können.

i) Kinder müssen sich verständlich ausdrücken können.

O

凶

j) Kinder müssen über einen sehr guten Wortschatz

凶 verfügen.

k) Kinder müssen grundlegende Fachbegriffe kennen.

O

凶

1) Kinder sollten sich trauen, vor anderen zu sprechen.

凶

\section{Kodierung:}

- Der Score bei Item 15 ergibt sich als Division der Summe der korrekt angekreuzten Antwortkategorien geteilt durch den Wert $\underline{3}$. Somit resultiert ein maximaler Score von 2 Punkten.

- Bei falsch angekreuzten Antwortkategorien erfolgt kein Punktabzug.

- Wurde keine der beiden Antwortoptionen ausgewählt: 0 Punkte für die betroffene Antwortkategorie.

16. Welche Unterstützungsmaßnahmen können Schülerinnen und Schülern das mündliche Vortragen erleichtern? Sie können sich für eine oder mehrere Antworten entscheiden.

a) Simulation/Vorbereitung in Kleingruppen

b) zur Verfügung stellen von sprachlichen Wendungen

c) Vortrag auswendig lernen lassen

d) Erstellen ausformulierter Moderationskarten

O

e) freie Themenwahl

f) Einüben kurzer Statements

Kodierung:

- Der Score bei Item 16 ergibt sich als Division der Summe der korrekt angekreuzten Antwortkategorien geteilt durch den Wert $\underline{3}$. Somit resultiert ein maximaler Score von 2 Punkten.

- Bei falsch angekreuzten Antwortkategorien erfolgt kein Punktabzug.

- Wurde keine der Antwortkategorien ausgewählt, beträgt der Score des Items 0 Punkte. 


\section{Worauf führen Sie die grammatikalischen Fehler des Zweitklässlers zurück?}

Sie können sich für genau eine Antwort entscheiden.

Noah berichtet Oliver in einem Brief aus dem Urlaub begeistert von seinem neuen Traumberuf:

„Lieber Oliver,

Ich will ein Meresbiologe sein! Ich war im Fischmuseum und da arbeiten viele Meresbiologen. Ein Meresbiologe erfoscht das Meer, die Pflantzen und die Vische die da leben. Er muss tauchen viele herum, damit er sie findet. Es ist ser gefärlich! Ein Hei ist ein ser große Visch. Über dem Wasser du siehst echt nur die Vlosse von dem Hei! Der Hei ist sehr schnell und kann fressen soga ein Mensch! Ich liebe Abenteur und wilde Tiere! Das ist warum ich ein super Meresbiologe bin!

Ich möchte jetzt wissen was du machest später

Dein Noah!“”

a) Noah leidet vermutlich an Legasthenie und hat daher sonderpädagogischen Förderbedarf.

O

b) Es handelt sich um typische Probleme beim Schriftspracherwerb im Anfangsunterricht.

O

c) Die Fehler lassen sich auf eine dialektal gefärbte Familiensprache zurückführen.

d) Der Schüler befindet sich im Zweitspracherwerb.

凶

e) Der Schüler könnte infolge einer Beeinträchtigung des Gehörs eine Sprachstörung aufweisen. Ein Facharzt sollte die Vermutung abklären.

\section{Kodierung:}

- Wurde die richtige Antwortkategorie in Item 17 angekreuzt, beträgt der Score 1 Punkt. Somit resultiert ein maximaler Score von 1 Punkt.

- Bei falsch angekreuzten Antwortkategorien erfolgt kein Punktabzug.

- Wurden mehrere oder keine Antwortkategorie(n) angekreuzt, beträgt der Score des Items 0 Punkte.

Maximal erreichbare Gesamtpunktzahl im FaWi-S: 34 Punkte 
3. Kodiermanual für qualitative Aufgaben 


\section{Kodier-Anweisungen zu Aufgabe 2a}

Aufgabe:

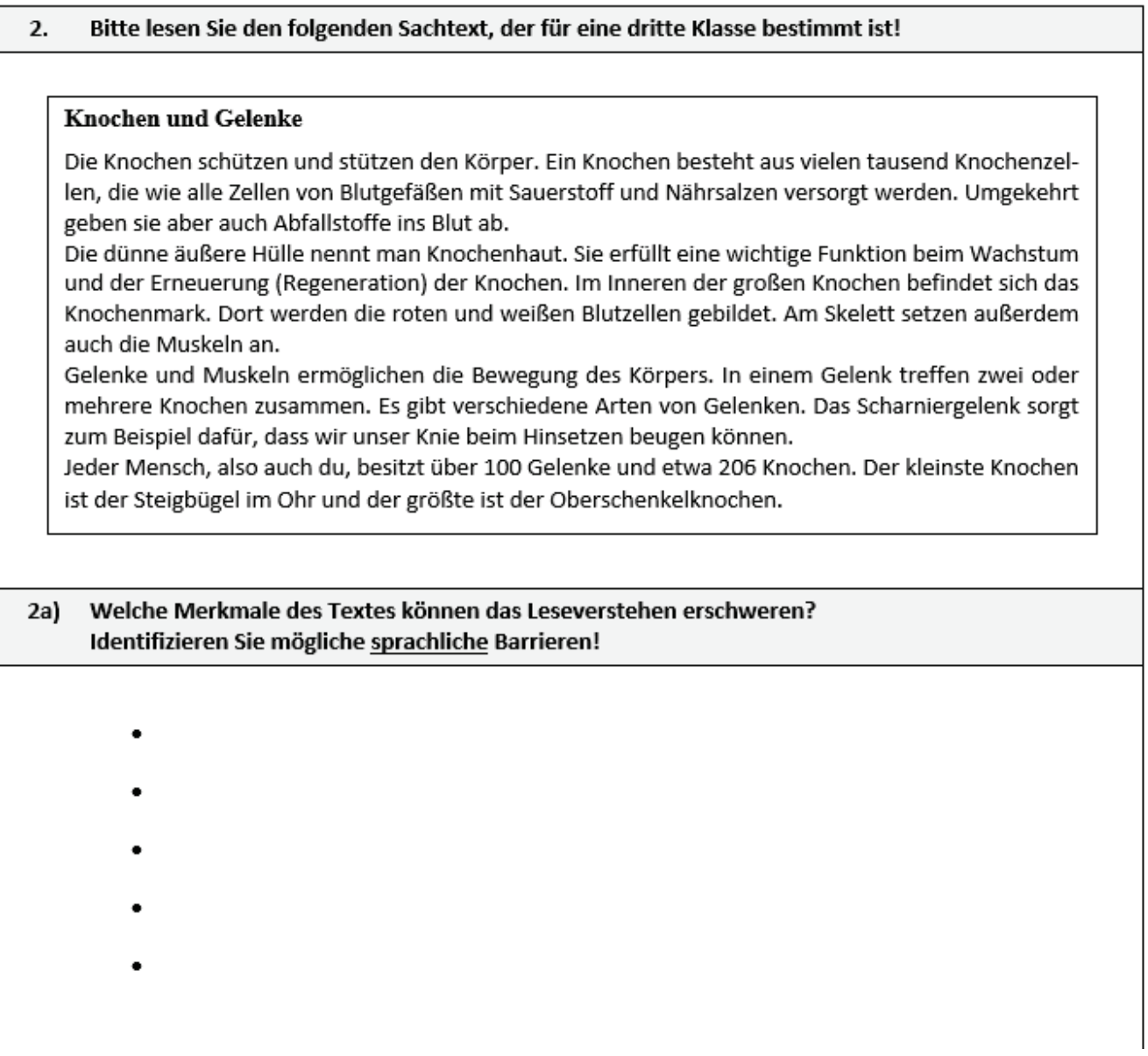

korrekt:

$\checkmark$ Fachwortschatz: fachsprachliche Lexik (z.B. Nährsalze, Scharniergelenk)

$\checkmark$ Fremdwörter: z.B. Regeneration

$\checkmark$ Mehrgliedrige Komposita: zusammengesetzte Namenwörter (z.B. Knochenzellen, Oberschenkelknochen)

$\checkmark$ Substantivierungen / Nominalisierungen

$\checkmark$ Bedeutungsdifferenzen (zwischen den Registern)

$\checkmark$ Relativsätze

$\checkmark$ Barrieren auf Verb-Ebene: zweiteilige Verben (geben ab, setzen an...), Passivformen

$\checkmark$ Kohäsive Mittel (Pronomen, Deiktika)

$\checkmark$ Informationsdichte

$\checkmark$ Keine grafische Hervorhebung von Schlüsselwörtern (z.B. Fettdruck)

$\checkmark$ Fehlende optische Entlastung (z.B. durch Bilder, Skizzen)

$\checkmark$ Keine Untergliederung mit Zwischenüberschriften

$\checkmark$ Worttrennung am Zeilenende / zeilenübergreifende Sätze 
ungültig: $\quad$ - Reines Aufzählen von Begrifflichkeiten aus dem Text

- Aspekte, die auf Satzlänge bzw. -komplexität abzielen (max. 20 Worte pro Satz, Nebensätze ersten Grades)

- Ungenaue Aussagen wie: Nicht altersentsprechend; Text ist nicht kindgerecht genug

- Aspekte, die auf Motivation oder Vorwissen der Lernenden abzielen (z.B. Aufbau des Textes nicht interessant / spannend für Kinder $\rightarrow$ kein sprachlicher Aspekt)

- Aspekte, die das Layout des Textes betreffen: Schriftgröße, Absatzstruktur, Blocksatz, Zeilenabstand (ausgenommen: keine grafische Hervorhebung von Schlüsselwörtern)

Kodierung:

- Der Score bei Item 2a ergibt sich als Division der Summe der korrekten Nennungen geteilt durch den Wert 2, wobei ein maximaler Score von 2 Punkten erreicht werden kann (= Deckelung).

- Bei falscher Nennung erfolgt kein Punktabzug.

- Wurde die Aufgabe nicht bearbeitet, beträgt der Score 0 Punkte. 
Kodier-Anweisungen zu Aufgabe 3

Aufgabe:

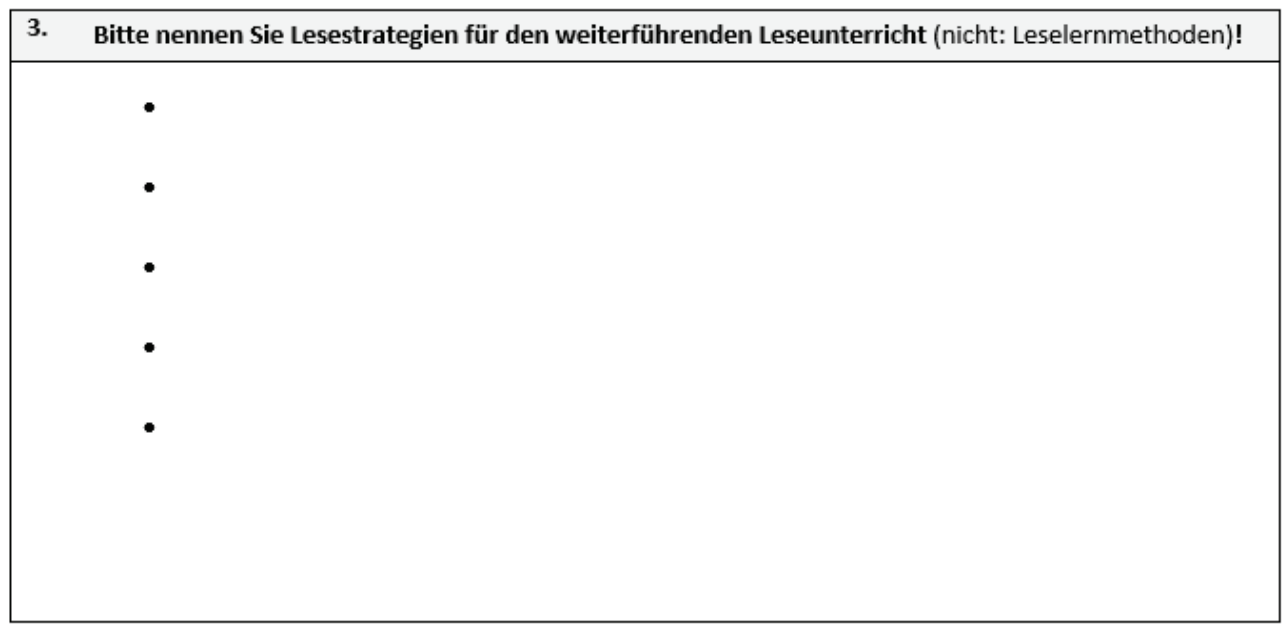

\section{Richtig:}

\section{$\checkmark$ Ordnende LS}

Ziel: Strukturierung und Reduktion des Textes auf

Kernaussagen

- Markieren von Schlüsselwörtern / Signalwörtern / wichtigen Textstellen

- Gliederung in Absätze

- (Teil-)Überschriften formulieren / zuordnen

- Visualisierung der Inhalte durch grafische Darstellungsformen (z.B. Mindmap, Cluster, Skizzen etc.)

- Überfliegendes Lesen / Scanning

- Anfertigen von Stichpunkten / Randnotizen

- Zusammengehörige Informationen verbinden

- Unwichtiges wegstreichen

\section{$\checkmark$ Elaborierende LS}

Ziel: Text wird mit Vorwissen, Gefühlen in Beziehung gesetzt

- Aktivierung des Vorwissens

- Ideensammlung anfertigen

- Markieren / Klärung unbekannter Begriffe

- Zunächst nur Überschrift lesen und Fragen an den Titel stellen

\section{$\checkmark$ Wiederholende LS}

Ziel: erneute Textauseinandersetzung, um Verstehens- und Behaltensleistung zu vertiefen

- Mehrmaliges Lesen

- Formulieren / Beantworten von Fragen zum Text 
- Nacherzählen / Zusammenfassen des Textes

- Abschreiben wesentlicher Passagen

$\checkmark$ Metakognitive LS

Ziel: Planung, Kontrolle, Anpassung des Strategieeinsatzes

$\checkmark$ Sammlungen von Lesestrategien: Lesenavigator, Lesepilot, 7-Schritt-Lesemethode, 5-Gang-Lesemethode

$\checkmark$ sich selbst etwas vorlesen (bei Unverständnis - im Sinne einer verständnisfördernden, individuell flexibel einsetzbaren Strategie)

Falsch:

- Ungenaue Angaben, die in Bewertung einen zu großen Spielraum lassen wie z.B. unterstreichen, nachschlagen

- Silbenlesen (nur für Anfangsunterricht gültig!)

- Lesen mit Lesehilfe, Leseschablone

- Lesen in verteilten Rollen; Rollenspiel zum Lesetext

- Leselernmethoden, wie z.B. 5-Gang-Leselernmethode,

- Tandemlesen, Lesen in Partnerarbeit

Kodierung:

- Der Score bei Item 3 ergibt sich als Division der Summe der korrekten Nennungen geteilt durch den Wert 3 , wobei ein maximaler Score von 2 Punkten erreicht werden kann (= Deckelung).

- Bei falscher Nennung erfolgt kein Punktabzug.

- Wurde die Aufgabe nicht bearbeitet, beträgt der Score 0 Punkte.

Quelle: Rosebrock (2017) 


\section{Literatur}

Sofern nicht anders angegeben, handelt es sich um selbstentwickelte Items, die auf folgenden Quellen fußen:

Bos, W., Hornberg, S., Arnold, K.-H., Faust, G., Fried, L., Lankes, E.-M., Schwippert, K. \& Valtin, R. (Hrsg.) (2007). IGLU 2006. Lesekompetenzen von Grundschulkindern in Deutschland im internationalen Vergleich. Münster: Waxmann.

Bredel, U. (2007). Sprachbetrachtung und Grammatikunterricht. Paderborn: UTB.

Fornol, S. (2019). Bildungssprachliche Mittel in Schülertexten aus dem Sachunterricht der Primarstufe. Landau in der Pfalz: Universität Koblenz-Landau, Dissertation.

Gibbons, P. (2002). Scaffolding language, scaffolding learning. Teaching second language learners in the mainstream classroom. Portsmouth NH: Heinemann.

Goldenstein, M. (i.V.). „Förderung der schriftlichen Erzählkompetenz von Grundschülern der 4. Jahrgangsstufe durch ein selbstreguliertes Lese- und Schreibtraining mit speziellem Fokus auf die textuellen Veränderungen bei leistungsstarken Kindern“, Regensburg: Dissertation.

Heilmann, B. (2019): Diagnostik \& Förderung - leicht gemacht. Herausgegeben von Prof. Wilhelm Grießhaber. 1. Auflage. Stuttgart: Ernst Klett Sprachen GmbH (Das Praxishandbuch).

Jeuk, Stefan (2018): Deutsch als Zweitsprache in der Schule. Grundlagen - Diagnose - Förderung. 4., überarbeitete und erweiterte Auflage. Stuttgart: Kohlhammer Verlag (Lehren und Lernen).

Koch, Peter; Oesterreicher, Wulf (1985): Sprache der Nähe - Sprache der Distanz. Mündlichkeit und Schriftlichkeit im Spannungsfeld von Sprachtheorie und Sprachgeschichte. In: Romanistisches Jahrbuch 36, S. 15-43.

Koch, Peter; Oesterreicher, Wulf (1994): Schriftlichkeit und Sprache. In: Hartmut Gunther und Otto Ludwig (Hg.): Schrift und Schriftlichkeit. Ein interdisziplinares Handbuch internationaler Forschung, Bd. 1. Berlin: De Gruyter (Handbücher zur Sprach- und Kommunikationswissenschaft HSK, 1), S. 587-604.

KMK (2004) = Sekretariat der Ständigen Konferenz der Kultusminister der Länder in der Bundesrepublik Deutschland. (2004). Bildungsstandards im Fach Deutsch für den Primarbereich. Neuwied: Luchterhand.

Li, M. (2017): Zweitsprachförderung im frühen naturwissenschaftlichen Lernen. Linguistisch hochwertige Formate und interaktive Elemente in der Unterrichtskommunikation. Weinheim: Beltz Juventa.

Morek, Miriam; Heller, Vivien (2012): Bildungssprache - Kommunikative, epistemische, soziale und interaktive Aspekte ihres Gebrauchs. In: Zeitschrift für angewandte Linguistik 57 (1), S. 67-101. DOI: 10.1515/zfal-2012-0011.

Pissarek, M. \& Schilcher, A. (2017): FALKO-D: Die Untersuchung des Professionswissens von Deutschlehrenden. Entwicklung eines Messinstruments zur fachspezifischen Lehrerkompetenz und Ergebnisse zu dessen Validierung. In Krauss, S., Lindl, A., Schilcher, A., Fricke, M., Göhring, A., Hofmann, B., Kirchhoff, P. \& Mulder, R. H. (Hrsg.). (2017). FALKO: Fachspezifische Lehrerkompetenzen. Konzeption von Professionswissenstests in den Fächern Deutsch, Englisch, Latein, Physik, Musik, Evangelische Religion und Pädagogik (S. 67 - 112). Münster: Waxmann. 
Quehl, T. (2010): Die Möglichkeiten des Scaffolding. Zur Gestaltung des Übergangs von der Alltagssprache der Kinder zur Fach- und Bildungssprache. In: Grundschulunterricht Deutsch (4), S. $28-32$.

Rosebrock, C. (2017): Grundlagen der Lesedidaktik und der systematischen schulischen Leseförderung. 8., korrigierte Aufl. Baltmannsweiler: Schneider-Verl. Hohengehren. 